\title{
Política de gasto real constante: efectos macroeconómicos de la composición del presupuesto y el superávit primario
}

\author{
Emerson Luís Lemos Marinho y Mauricio Benegas
}

\section{Resumen}

En este artículo se analiza la política fiscal de gasto real constante adoptada recientemente por las autoridades fiscales del Brasil. También se compara la política de mantener un superávit primario como proporción del producto interno bruto con la de modificar la composición del gasto en favor de la inversión, a fin de determinar cuál de las dos políticas es más eficiente para promover el crecimiento económico. Se investigan los efectos de estas políticas en el consumo, la inversión, la oferta de mano de obra y la producción a largo y corto plazo, y la reacción de la estructura temporal de los tipos de interés. También se analiza la relación entre estas políticas fiscales y el bienestar. Se utiliza un modelo de agente representativo de maximización de la utilidad intertemporal sujeto a restricciones presupuestarias, con una previsión perfecta y un horizonte infinito.

\section{Palabras clave}

Política fiscal, gastos públicos, condiciones económicas, consumo, inversiones, tasas de interés, bienestar social, análisis económico, Brasil

\section{Clasificación JEL}

$$
\text { E1, E2, H3 }
$$

\section{Autores}

Emerson Luís Lemos Marinho es Profesor del Departamento de Finanzas de la Universidad Federal de Ceará (UFC) (Brasil). Correo electrónico: emarinho@ufc.br.

Mauricio Benegas es Profesor del Departamento de Finanzas de la Universidad Federal de Ceará (UFC) (Brasil). Correo electrónico: mauricio_benegas@caen.ufc.br. 


\section{Introducción}

En los últimos años, el Brasil se ha enfrentado a una de sus peores recesiones desde que comenzó a medirse el producto interno bruto (PIB) en el país. Además, el déficit presupuestario del gobierno ha empeorado con el tiempo, lo que significa que el país puede tener serias dificultades para financiar este déficit si no se adoptan medidas urgentes.

El análisis estadístico muestra que durante el período de crecimiento significativo del déficit primario, los principales indicadores socioeconómicos del país empeoraron sustancialmente. Según datos del Instituto de Investigaciones Económicas Aplicadas (IPEA, 2016), de julio de 2011 a septiembre de 2016, las cuentas primarias cayeron bruscamente de un superávit del $3,57 \%$ del PIB a un déficit del $3,05 \%$ del PIB. La tasa de crecimiento del PIB real del 3,4\% en el tercer trimestre de 2011 se redujo al $-4,84 \%$ en el cuarto trimestre de 2015 . En el contexto de esta recesión, la inflación aumentó del $6,08 \%$ al $11,28 \%$ entre 2011 y 2015.

Esta situación pesó mucho en las condiciones económicas de las familias. La tasa de desempleo, por ejemplo, aumentó del 7,9\% en marzo de 2013 al 13,7\% en marzo de 2017 y la masa salarial real se contrajo aproximadamente 10.000 millones de reales entre 2015 y 2016.

Con el fin de abordar este problema, las autoridades fiscales adoptaron recientemente una política de gasto público real constante para los próximos años, según la cual el gasto nominal para un año determinado será, como máximo, igual al del año anterior más la inflación en ese período.

Teniendo en cuenta las dificultades que se enfrentan, el presente artículo tiene por objeto analizar las posibles consecuencias de esta política en las trayectorias de equilibrio del consumo, la inversión y la producción a largo y corto plazo, así como la reacción de la estructura temporal de los tipos de interés. El análisis de esta última es primordial, dada su importancia en la transmisión de las políticas macroeconómicas.

Sin embargo, algunos economistas brasileños han sostenido que las autoridades fiscales también deberían adoptar una política de mantenimiento de un superávit primario como proporción del PIB, a fin de hacer frente al déficit fiscal y acelerar el crecimiento económico de inmediato. Al mismo tiempo, otros economistas brasileños creen que el gobierno debería modificar su composición presupuestaria para reflejar una mayor inversión en infraestructura que en gasto ${ }^{1}$. Esta infraestructura permitiría a las empresas privadas aumentar su productividad, contribuyendo así a un crecimiento económico más rápido.

El objetivo principal de este artículo es determinar, partiendo del supuesto de que el gasto público total en términos reales se mantiene constante, cuál de las dos políticas fiscales sugeridas es más eficaz para producir mayores niveles de producción e inversión a largo plazo y, en última instancia, producir un mayor crecimiento económico.

Varios estudios se han centrado en el impacto de las políticas fiscales en la estructura temporal de los tipos de interés. En cuanto a la utilización del enfoque tradicional de los modelos de IS-LM con algunas variaciones, cabe mencionar los trabajos de Blanchard (1981), Turnovsky y Miller (1984) y McCafferty (1986). En un contexto diferente que considera el comportamiento de optimización intertemporal de un agente representativo, cabe mencionar también los artículos de Cox, Ingersoll y Ross (1985) y Fisher y Turnovsky (1992).

Este artículo se basa en el modelo de Fisher y Turnovsky (1992) con dos modificaciones principales. La primera asume que el gasto real del gobierno es constante a través del tiempo. La segunda sugiere que la política fiscal adoptada por el gobierno es de superávit primario como porcentaje del PIB. Este modelo de agente representativo emplea la maximización de la utilidad intertemporal con sujeción a restricciones presupuestarias, con una previsión perfecta y un horizonte infinito.

\footnotetext{
1 Baxter y King (1993) calibran un modelo de ciclo económico real para derivar la respuesta dinámica de transición del consumo, la inversión y el empleo a los cambios en la composición del gasto público.
} 
Dado que los activos de las carteras financieras pueden ajustarse instantáneamente y sin costo alguno, los resultados económicos reales son independientes del tipo de interés a largo plazo, mientras que el equilibrio a corto plazo determina el tipo de interés a corto plazo. Como resultado, el tipo de interés a largo plazo se establece mediante el arbitraje entre los equilibrios a corto y largo plazo. Por lo tanto, el tipo de interés a corto plazo responde a los choques de la política fiscal inmediatamente, mientras que el tipo a largo plazo responde indirectamente a través de los efectos de las expectativas actuales y futuras de los tipos de interés a corto plazo.

En esta situación, se considerarán dos tipos de choque de política fiscal: el primero es un choque permanente no previsto por los agentes; en otras palabras, un shock inmediato. El segundo es un choque permanente que se anticipa en el futuro. En este caso, el gobierno anuncia que modificará la política fiscal en algún momento del futuro. Además del supuesto de que el gasto público real se mantendrá constante a lo largo del tiempo, se analizarán dos medidas de política fiscal. La primera examina la forma en que los cambios en la composición del gasto público (por ejemplo, un mayor gasto público en consumo o en infraestructura) afectan a la inversión, el consumo privado, la producción, la oferta de mano de obra y los tipos de interés a largo y corto plazo. La segunda medida, alineada con la primera, analiza el impacto del crecimiento del superávit primario como porcentaje del PIB.

En este artículo también se analiza la relación entre estas políticas fiscales y el bienestar. El modelo se ha construido según ciertas especificaciones para obtener resultados objetivos. En particular, se supone que la función de utilidad representativa sigue la especificación adoptada por Christiano, Eichenbaum y Rebelo (2011) y que la tecnología aplicada se representa por una función de producción Cobb-Douglas para el gasto público, como sugiere Barro (1990). Con estas especificaciones, las políticas de austeridad fiscal y de composición del presupuesto que maximizan el bienestar social se miden a través de la utilidad total descontada. Por lo tanto, se demuestra que, en condiciones razonables, una composición presupuestaria que maximice el bienestar debería asignar la mayor parte de los recursos a la infraestructura y que la proporción del PIB asignada al superávit primario depende de la sensibilidad del ingreso per cápita a los resultados fiscales.

Incluida la introducción, este artículo está dividido en seis secciones. En la sección ll se presenta y considera el modelo teórico aplicado. En la sección III se analiza la dinámica del equilibrio transaccional cuando el gobierno anuncia cambios en la composición de su gasto y aumenta el superávit primario como proporción del PIB. En la sección IV se examinan los efectos de esas políticas fiscales en la estructura temporal de los tipos de interés. En la sección $\vee$ se extraen algunas conclusiones sobre el bienestar de los agentes cuando el gobierno altera la composición de su gasto y aumenta su superávit primario como porcentaje del PIB. Por último, en la sección VI se presentan las conclusiones. También hay un anexo que muestra formalmente las soluciones del modelo y algunas de las ecuaciones algebraicas aplicadas para obtener resultados.

\section{El modelo teórico}

El modelo considera una economía cerrada ${ }^{2}$ en la que agentes idénticos que se supone que tienen una vida infinita toman decisiones inmediatas sobre el consumo $c(t)$ y el trabajo $l(t)$, buscando la maximización intertemporal de los flujos de utilidad futuros $U\left(c(\mathrm{t}), l(t), g_{c}(t)\right)$ descontados a una tasa $\rho$. El término $g_{c}(t)$ representa el gasto de consumo del gobierno. La función de utilidad instantánea del agente se define en la forma aditiva como: $u(c(t), l(t))+v\left(g_{c}(t)\right.$, en que $u$ and $v$ son formas estrictamente cóncavas con las siguientes propiedades: $u_{c}>0, u_{c c}<0, u_{l}<0, u_{l l}<0, v^{\prime}>0$ y $v^{\prime \prime}<0^{3}$.

\footnotetext{
2 Véase en Agénor (2005) un modelo basado en una economía abierta.

3 Véase la justificación de este enfoque en Aschauer (1988, 1990 y 2008).
} 
Siguiendo a Barro (1990), la función de producción se definirá en la forma multiplicativa como: $y(t)=f\left(k(t), l(t) h\left(g_{i}(t)\right)\right.$, donde $y(t)$ es la producción real, $k(t)$ es el acervo de capital físico, $l(t)$ es la oferta de mano de obra y $g_{i}(t)$ es el gasto de infraestructura del gobierno. La función de producción es neoclásica con las siguientes propiedades: $f$ es linealmente homogénea con $f_{k}>0, f_{l}>0, f_{k k}<0$, $f_{l l}<0$ y $h^{\prime}>0, h^{\prime \prime}<0$.

En principio, el gasto público en infraestructura ejerce una externalidad positiva sobre la productividad marginal del capital privado. De hecho, la productividad marginal del capital privado viene dada por $f_{k} h\left(g_{j}\right)$. Dado que $h$ es una función cada vez más importante del gasto en infraestructura, un aumento de la infraestructura eleva la productividad marginal del capital. La intensidad de este efecto externo positivo depende de la especificación de la función de producción. En general, los efectos marginales del capital privado y del gasto en infraestructura de producción dependen una vez más de la especificación de la función de producción y del vínculo entre los parámetros y $k$ y $g$.

Teniendo en cuenta la restricción presupuestaria, $y(t)=f\left(k(t), l(t) h\left(g_{i}(t)\right)=c(t)+i(t)+g(t)\right.$, por lo tanto, el acervo de capital evoluciona de acuerdo con la siguiente identidad macroeconómica:

$$
\dot{k}(t)=f\left(k(t), l(t) h\left(g_{i}(t)\right)-c(t)-g(t)\right.
$$

donde $g(t)$ representa el total de los gastos reales del gobierno e $i(t)=\dot{k}(t)$. El término $k(t)$ representa el derivado temporal de $k(t)$.

La restricción presupuestaria a la que se enfrenta el gobierno está representada por la ecuación diferencial:

$$
\dot{b}(t)=r b(t)+g(t)-T(t)
$$

donde $b(t)$ es el acervo de deuda pública, $T(t)$ es la suma global de ingresos fiscales, $r$ es el tipo de interés real y $\dot{b}(t)$ el derivado de $b(t)$ respecto al tiempo.

El total de los gastos reales del gobierno se considerará constante a lo largo del tiempo de acuerdo con la regla de política fiscal adoptada por el gobierno. Dicho esto, el gasto total se distribuirá entre el gasto de consumo del gobierno $-g_{c}(t)-$ y el gasto de infraestructura $-g_{i}(t)$. En otras palabras, $\bar{g}=g_{c}(t)+g_{i}(t)$, donde $\bar{g}$, representa el gasto total constante asumiendo que $g_{c}(t)=\alpha \bar{g}$ y que $g_{i}(t)=(1-\alpha) \bar{g}$ donde $0<\alpha<1$.

Además, el ajuste de la política fiscal adoptado por el gobierno será el superávit primario como porcentaje del PIB, lo que significa que:

$$
T(t)-g(t)=\beta y(t)=\beta f\left(k(t), l(t) h\left(g_{i}(t)\right)\right.
$$

donde $T(t)-g(t)$ representa el superávit primario y $0<\beta<1$.

Por lo tanto, el problema del agente representativo se resume formalmente como:

$$
\max _{\{c, l\}} \int_{0}^{\infty} e^{-\rho}\left[u(c(t), l(t))+v\left(g_{c}(\alpha \bar{g})\right] d t\right.
$$

sujeto a restricciones:

$$
\dot{k}(t)+\dot{b}(t)=(1-\beta) f\left(k(t), l(t) h\left((1-\alpha \bar{g})+r b(t)-c(t)-\bar{g}, k(0)=k_{0} b(0)=b_{0} .\right.\right.
$$

Obsérvese que esta última restricción se genera después de la manipulación algebraica de las expresiones (1), (2) y (3) y de las definiciones de $g_{i}(t)$ y $g_{c}(t)$. Las dos últimas restricciones suponen que en el estado inicial, la economía tiene un acervo de capital $k_{0}$ y su deuda pública es igual a $b_{0}$.

Las condiciones hamiltonianas de primer orden de valor actual asociadas al problema (4) están dadas por:

$$
u_{c}(c(t), l(t))=\lambda(t)
$$




$$
\begin{gathered}
u_{l}(c(t), l(t))=-\lambda(t)(1-\beta) f(k(t), l(t) h((1-\alpha) \bar{g}) \\
\lambda(t)(1-\beta) f_{k}(k(t), l(t) h(1-\alpha) \bar{g})=\rho \lambda(t)-\dot{\lambda}(t) \\
\lambda(t) r=\rho \lambda(t)-\dot{\lambda}(t) \\
\lim _{t \rightarrow \infty} \lambda(t) e^{-\rho t} k(t)=0 \\
\lim _{t \rightarrow \infty} \lambda(t) e^{-\rho t} b(t)=0
\end{gathered}
$$

donde $\lambda(t)$ es la utilidad marginal actual de la riqueza. Las condiciones de transversalidad (9) y (10) se establecen de manera que las trayectorias de $(t)$ and $k(t)$ no sean explosivas.

\section{Análisis de equilibrio a corto plazo}

Las soluciones dirigidas al consumo $c(t)$ y la oferta de mano de obra $l(t)$, así como la utilidad marginal de las funciones de riqueza $\lambda(t)$ del acervo de capital $k(t)$ y de los parámetros de política $\alpha$ y $\beta$, se obtienen a partir de las ecuaciones (5) y (6). Por lo tanto, obtenemos las soluciones $c=c(\lambda, k, \alpha, \beta)$ y $l=l(\lambda, k, \alpha, \beta)$, en las que el período de tiempo $t$ ya no se incluye como argumento de la función para los efectos de simplificación de la notación. Para analizar el efecto de cada uno de los términos $\lambda, k, \alpha$ y $\beta$ en $c$ y $l$, utilizamos los sistemas de ecuaciones (5) y (6). La solución de este sistema proporciona los signos para las siguientes relaciones: $\frac{\partial c}{\partial k}=c_{k}<0, \frac{\partial c}{\partial \lambda}=c_{\lambda}<0, \frac{\partial c}{\partial \alpha}>0, \frac{\partial c}{\partial \beta}=c_{\beta}>0, \frac{\partial l}{\partial k}=l_{k}>0$, $\frac{\partial l}{\partial \lambda}=l_{\lambda}>0, \frac{\partial l}{\partial \alpha}=l_{\alpha}<0$ y $\frac{\partial l}{\partial \beta}=l_{\beta}<0$.

En el anexo se muestra formalmente cómo se obtienen las expresiones y los respectivos signos de las ecuaciones anteriores. El impacto a corto plazo de las variaciones del acervo de capital y la utilidad marginal de la riqueza en el consumo y la oferta de mano de obra son como se esperaba. Mientras tanto, de acuerdo con las expresiones de $\frac{\partial c}{\partial k}$ y $\frac{\partial l}{\partial k}$ obtenidas en las soluciones del sistema, cuando el superávit primario como porcentaje del PIB aumenta (crecimiento de $\beta$ ), tanto el aumento del consumo privado como la disminución de la oferta de mano de obra se desaceleran.

El aumento del gasto público destinado al consumo (crecimiento de $\alpha$ ) implica un aumento del consumo privado y una disminución de la oferta de mano de obra. Estos dos resultados son menos sensibles a los aumentos del porcentaje del PIB asignado al superávit primario (crecimiento de $\beta$ ).

Por último, el aumento del porcentaje de superávit primario da lugar a un aumento del consumo privado y a una disminución de la oferta de mano de obra. En lo que respecta al aumento del consumo privado, el incremento de $\beta$ afecta al consumo mediante un efecto ingreso y un efecto de sustitución predominante. En consecuencia, las expresiones (6) y (7) dan lugar a la expresión $r=(1-\beta) f_{k} k$. En estos términos, $d r / d \beta<0$. Por lo tanto, el crecimiento de $\beta$ desencadena una caída de los tipos de interés, lo que hace que las personas reduzcan sus ahorros y aumenten el consumo privado.

\section{Análisis de equilibrio a largo plazo}

La dinámica de equilibrio de estado estacionario se obtiene sustituyendo las soluciones de las funciones $c=c(\lambda, k, \alpha, \beta)$ y $l=l(\lambda, k, \alpha, \beta)$ en las ecuaciones diferenciales (5) y (6). Por lo tanto, obtenemos el siguiente sistema de ecuaciones diferenciales:

$$
\begin{gathered}
\dot{k}=f(k, l(\lambda, k, \alpha, \beta)) h((1-\alpha) \bar{g})-c(\lambda, k, \alpha, \beta)-\bar{g} \\
\dot{\lambda}=\left[\rho-(1-\beta) f_{k}(k(\lambda, k, \alpha, \beta), l(\lambda, k, \alpha, \beta)) h((1-\alpha) \bar{g})\right] \lambda
\end{gathered}
$$


Aplicando $\dot{k}=\dot{\lambda}=0$ en el sistema anterior, podemos obtener el equilibrio de estado estacionario. Por lo tanto, tenemos que $f(\widetilde{k}, l(\tilde{\lambda}, \widetilde{k}, g, \alpha, \beta)) h((1-\alpha) \bar{g})=c(\tilde{\lambda}, \widetilde{k}, g, \alpha, \beta)+\bar{g}$ y $(1-\beta) f_{k}(\tilde{k}, l(\tilde{\lambda}, \tilde{k}, g, \alpha, \beta)) h((1-\alpha) \bar{g})=\rho$, donde la solución del sistema anterior ayuda a establecer las variables de equilibrio. Las variables con una tilde $(\sim)$ representan valores en equilibrio estacionario. Los impactos a largo plazo de los parámetros de política fiscal $\alpha$ y $\beta$ en el acervo de capital y la utilidad marginal a largo plazo de la riqueza están dados por las siguientes relaciones ${ }^{4}: \partial \widetilde{k} / \partial \alpha<0$, $\partial \widetilde{k} / \partial \beta<0, \partial \tilde{\lambda} / \partial \alpha>0$ y $\partial \widetilde{\lambda} / \partial \beta>0$.

Por lo tanto, los aumentos de $\alpha$ y $\beta$ reducen el acervo de capital y mejoran la utilidad marginal de la riqueza (reducción del consumo privado a largo plazo).

El análisis de la dinámica del sistema es más fácil después de la linealización de las ecuaciones (11) y (12) en torno a su equilibrio de estado estacionario. En consecuencia, obtenemos la siguiente dinámica:

$$
\begin{gathered}
\dot{k}=w_{11}(k-\widetilde{k})+w_{12}(\lambda-\tilde{\lambda}) \\
\dot{\lambda}=-\tilde{\lambda}(1-\beta)_{w_{21}}(k-\tilde{k})-\tilde{\lambda}(1-\beta) w_{22}(\lambda-\tilde{\lambda})
\end{gathered}
$$

donde $w_{11}=\left(f_{k} l_{k} h-c_{k}\right)>0, w_{12}=\left(f_{l} l_{\lambda} h-c_{\lambda}\right)>0, w_{21}=\left(f_{k k}+f_{k} l_{k}\right) h<0$ y $w_{22}=f_{k} l_{k} h>0$.

En el anexo se muestra formalmente que el equilibrio a largo plazo es del tipo de punto de silla y que las ecuaciones de brazo estable e inestable están representadas respectivamente por: $\lambda=\tilde{\lambda}+\frac{\left(\mu_{1}-w_{11}\right)}{w_{12}}(k-\tilde{k})$ y $\lambda=\tilde{\lambda}+\frac{\left(\mu_{2}-w_{11}\right)}{w_{12}}(k-\tilde{k})$, donde $\mu_{1}$ y $\mu_{2}$ son valores propios de las matrices del sistema de ecuaciones (13) y (14). Dado que el equilibrio es un tipo de punto de silla, se asume, sin pérdida de generalidad, que $t \mu_{1}<0$ y $\mu_{2}>0$.

Como se muestra en el anexo, la línea recta del brazo estable presenta una inclinación negativa mientras que la del brazo inestable tiene una inclinación positiva pero menor que la del brazo estable.

Diseñando estas líneas rectas en el espacio $\lambda \times k$ considerando las características de equilibrio del punto de silla y las propiedades de los brazos estables e inestables, se obtiene el diagrama de fase de este sistema como se muestra en el gráfico 1. Las líneas rectas SS y NN representan el brazo estable y el brazo inestable, respectivamente.

Gráfico 1

Diagrama de fase del sistema

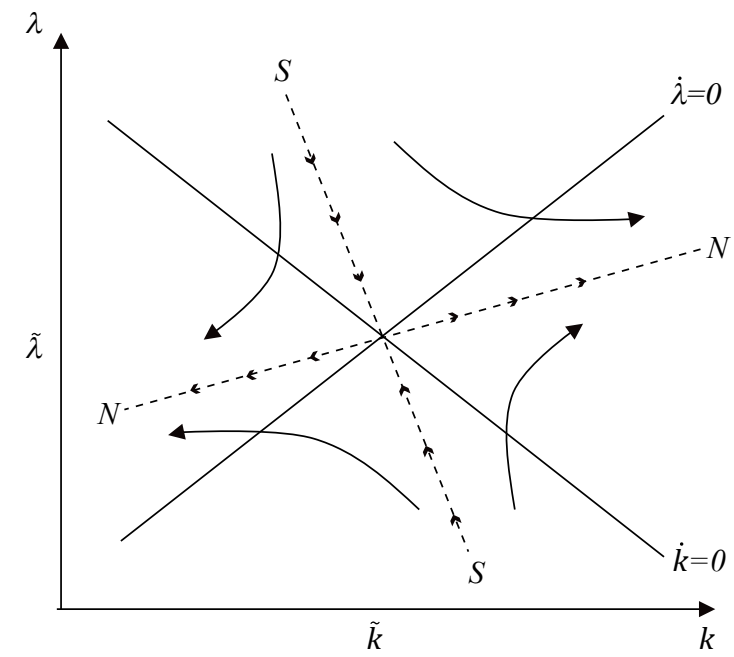

Fuente: Elaboración propia.

\footnotetext{
4 Estas relaciones de largo plazo se obtienen solucionando los sistemas de ecuaciones $\dot{\lambda}=0$ y $\dot{k}=0$.
} 


\section{Choques fiscales permanentes previstos y no previstos}

En esta sección se analizan las consecuencias de dos casos de choque fiscal. En el primero, el gobierno anuncia que aumentará el gasto en infraestructura en detrimento del gasto de consumo del gobierno. Esto implica una reducción del parámetro de política fiscal $\alpha$. En el segundo caso, el gobierno anuncia una política de crecimiento del superávit primario como porcentaje del PIB. Esto resulta en un aumento del parámetro $\beta$.

En ambos casos, se considera que estos anuncios de política fiscal no eran previstos; el cambio de política tiene lugar inmediatamente. Cuando se anticipan cambios de política, el gobierno anuncia que tendrán lugar en un futuro tiempo $T$. Además, los choques fiscales son permanentes.

El objetivo principal es identificar cuál de las dos políticas fiscales es mejor para el crecimiento económico. Los objetivos secundarios son analizar los efectos de esos cambios en las trayectorias del acervo de capital $k(t)$, la utilidad marginal de la riqueza $\lambda(t)$ y la estructura temporal de los tipos de interés. El anexo incluye una descripción formal de las soluciones del modelo y de cómo se producen los desplazamientos de equilibrio.

\section{Choque fiscal permanente en $\alpha$}

Inicialmente, se considera la política fiscal no prevista de aumento de los gastos de infraestructura (reducción de $\alpha$ ) anunciada por el gobierno en el presente. Ya que $\partial \widetilde{k} / \partial \alpha<0$ y $\partial \widetilde{\lambda} / \partial \alpha>0$, el nuevo equilibrio a largo plazo tendrá un mayor acervo de capital (mayores niveles de inversión) y una menor utilidad marginal de la riqueza, lo que implica un mayor consumo privado que el equilibrio inicial.

Obsérvese en el gráfico 2A que el anuncio inmediato de una reducción en $\alpha$ causa desplazamientos en las líneas del brazo estable XX y del brazo inestable YY. Ambas rectas se desplazan hacia la derecha, originando un nuevo equilibrio de estado estacionario representado por el punto C. Por lo tanto, hay un desplazamiento del equilibrio inicial A a un nuevo equilibrio de largo plazo C.

Gráfico 2

A. Choque no previsto

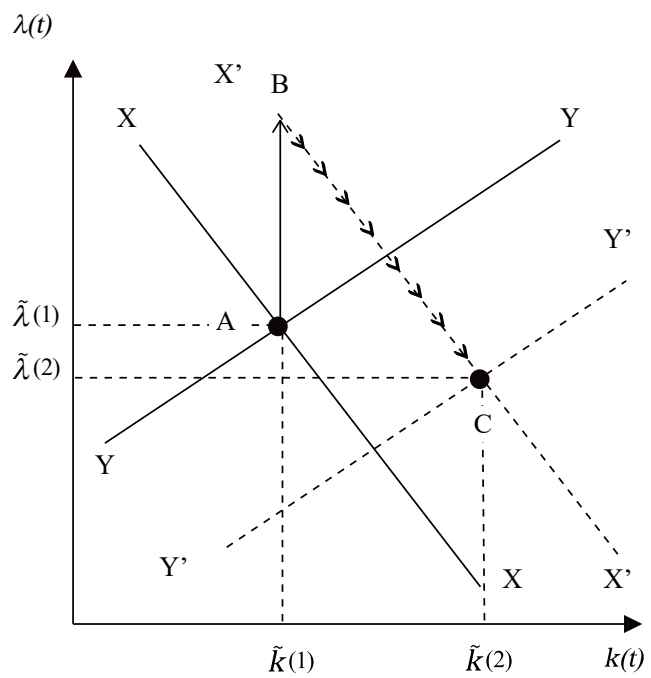

B. Choque previsto

$\lambda(t)$

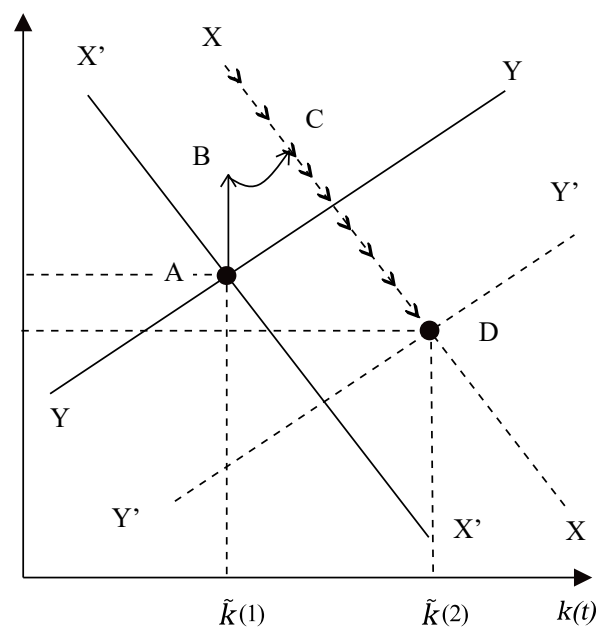

Fuente: Elaboración propia. 
A corto plazo, justo después del anuncio de una reducción en $\alpha$, la utilidad marginal de la riqueza $\lambda(0)$ salta directamente al brazo estable (punto $B$ en la línea recta $X^{\prime} X^{\prime}$ ). Esto da lugar a una disminución de la riqueza privada, lo que provoca una reducción del consumo privado y un aumento de la oferta de mano de obra. El aumento de la oferta de mano de obra conduce a una mejora de la productividad marginal del capital, lo que se traduce en un aumento del tipo de interés a corto plazo.

Al mismo tiempo, la productividad marginal del capital aumenta más que la del equilibrio de estado estacionario. De acuerdo con la ecuación de equilibrio (7), esto implica que $\dot{\lambda}(t)<0$, lo que supone que la utilidad marginal de la riqueza debería disminuir, mientras que el consumo privado debería crecer. Sin embargo, obsérvese que el tiempo empleado por ambos para alcanzar un nuevo equilibrio de estado estacionario según la ecuación (14) aumenta a medida que aumenta el superávit primario como porcentaje del PIB (mayor para $\beta$ ).

Todavía puede observarse que la caída de los niveles iniciales de consumo privado mejora el ahorro, lo que da lugar a una acumulación de capital físico. En este contexto, la economía sigue una trayectoria de B a C. El consumo privado, el acervo de capital (inversión), la producción y la oferta de mano de obra son mayores en este nuevo equilibrio que en el equilibrio inicial.

En el caso de un choque previsto, cuando el gobierno anuncia una reducción de $\alpha$ en un fecha futura $T$, siempre que los agentes descuenten el cambio en el futuro, el salto en la utilidad marginal de la riqueza debería ser menor. En el anexo se muestra que cuanto más lejano sea el momento del cambio de política, más bajo será el salto $\lambda(t)$. Por lo tanto, $\lambda(t)$ salta de $\mathrm{A}$ a $\mathrm{B}$, como se muestra en el gráfico $2 \mathrm{~B}$.

Como en el caso no previsto, el acervo de capital en el nuevo equilibrio será mayor y la utilidad marginal de la riqueza será menor, y, en consecuencia, el consumo privado aumentará. La principal diferencia es el salto más bajo $\lambda(0)$, que resulta en una menor disminución de la riqueza privada. Por lo tanto, la oferta de mano de obra aumenta mientras que la producción crece y el consumo privado disminuye, pero en menor medida.

A corto plazo, los efectos adversos de una política prevista son más limitados que en el caso de la misma política anunciada inmediatamente. Por ejemplo, la reducción del consumo en el primer caso es más limitada que en el segundo. Por lo tanto, parece aconsejable que el gobierno adopte políticas previstas a corto plazo.

Al mismo tiempo, a medida que aumenta la oferta de mano de obra, la productividad marginal del capital mejora, lo que hace que aumenten los tipos de interés a corto plazo. Esto también hace que $\dot{\lambda}(t)>0$, en línea con la ecuación de equilibrio (7). Este resultado, combinado con el aumento del ahorro, hace $\lambda(t)$ y $k(t)$ sigan la trayectoria inestable BC hasta llegar al brazo estable $X^{\prime} X^{\prime}$. De hecho, el cambio $\alpha$ tiene lugar en el período $T$. El desplazamiento de $\lambda(t)$ y $k(t)$ en esta trayectoria se muestra en el gráfico 2A.

A partir de este punto, $\lambda(t)$ y $k(t)$ siguen sus trayectorias en el brazo estable hasta alcanzar el nuevo equilibrio a largo plazo, representado por el punto $D$ en el gráfico 2B. El consumo privado, el acervo de capital (inversión) y la producción también son mayores en comparación con el equilibrio inicial.

Obsérvese también que los efectos de un aumento del gasto de consumo del gobierno en el consumo privado, la inversión y la producción no son simétricos a los que se producen en el caso de un aumento del gasto en infraestructura, independientemente de que se prevea o no el anuncio.

\section{Choque fiscal permanente en $\beta$}

En este caso, el gobierno anuncia en el presente (política fiscal no prevista) que aumentará el superávit primario como porcentaje del PIB (aumento en $\beta$ ). Según los signos $\partial \widetilde{k} / \partial \beta<0$ y $\partial \widetilde{\lambda} / \partial \beta>0$ en el escenario de equilibrio a largo plazo, el acervo de capital será inferior a la utilidad marginal de la riqueza 
en comparación con el equilibrio inicial. De hecho, en el gráfico 2C, el aumento en $\beta$ desplaza el brazo estable de XX a X'X' y el brazo inestable de YY a Y'Y'. El nuevo equilibrio de estado estacionario (largo plazo) está representado por el punto C.

\section{Gráfico 2}

C. Choque no previsto

$\lambda(t)$

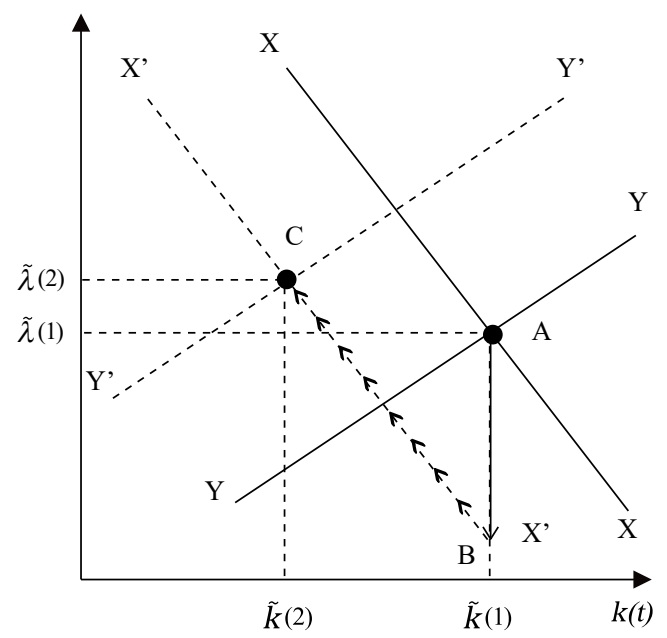

D. Choque previsto

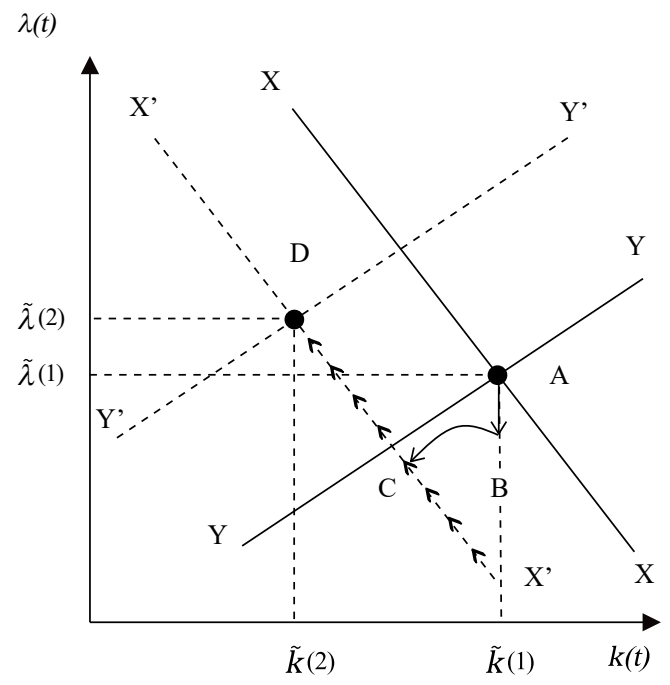

Fuente: Elaboración propia.

Tras el anuncio, la utilidad marginal de la riqueza $\lambda(0)$ salta inmediatamente de un equilibrio inicial A y alcanza el brazo estable X'X' en el punto B. El crecimiento de la riqueza privada debido a la disminución de $\lambda(t)$ es a corto plazo y da lugar a una reducción de la oferta de mano de obra, lo que a su vez provoca una disminución de la productividad marginal del capital y, por tanto, de los tipos de interés a corto plazo.

Al mismo tiempo, la disminución de la productividad marginal del capital por debajo del equilibrio a largo plazo hace que la utilidad marginal de la riqueza vuelva a crecer para mantener la ecuación de equilibrio (7). A esto le sigue una caída del consumo privado. Mientras tanto, la mejora inicial del consumo (disminución del ahorro) genera una reducción del capital físico. En este contexto, las trayectorias de $\lambda(t)$ y $k(t)$ se mueven desde el punto B a un nuevo equilibrio a largo plazo representado por el punto C. En este nuevo equilibrio a largo plazo, el consumo privado, el acervo de capital físico y la producción son menores y la oferta de mano de obra es mayor en comparación con el equilibrio inicial.

Los efectos del anuncio de un cambio de política que se producirá en una fecha futura $T$ (política fiscal prevista) son similares a los del caso de un anuncio no previsto. La principal diferencia es que el salto de $\lambda(0)$ es menor porque los agentes anticipan que los cambios de política solo se producirán en un tiempo futuro $T$.

A corto plazo, las políticas previstas producen menos efectos adversos que las políticas no previstas. Por ejemplo, la disminución del consumo privado cuando se aplica una política prevista es menor que cuando se aplica una política no prevista. Este resultado indica que las políticas previstas a corto plazo son preferibles a las políticas no previstas.

Según el gráfico 2D, en el caso de un choque previsto, la utilidad marginal de la riqueza cae de A a B, lo que significa que la riqueza privada debería crecer. Cuanto más alto es el valor $T$, más bajo es el salto $\lambda(0)$. A corto plazo, la productividad marginal del capital disminuye, lo que implica una caída de los tipos de interés a corto plazo, pero en menor medida. Considerando la ecuación de equilibrio 
(7) podemos concluir que la caída de la productividad marginal del capital implica que $\dot{\lambda}(t)>0$. En consecuencia, el consumo privado debe disminuir para mantener la utilidad marginal del equilibrio de la riqueza. Al mismo tiempo, el crecimiento del consumo inicial genera una reducción del ahorro, desalentando así las inversiones. Por lo tanto, la economía sigue una trayectoria de C a D, cuando alcanza un nuevo equilibrio a largo plazo.

En este nuevo equilibrio, el consumo privado, el acervo de capital, la producción y la oferta de mano de obra son similares a los niveles observados en casos no previstos cuando se comparan los equilibrios inicial y final. Los efectos de un recorte del superávit primario (caída en $\beta$ ) son claramente simétricos a los efectos de un aumento del superávit primario.

En resumen, en términos de generación de crecimiento económico, la política fiscal de gasto en infraestructura es más eficaz que la política de mantenimiento de un superávit primario como proporción del PIB. Por lo tanto, esta política de gasto en infraestructura, combinada con la política de gasto real constante, es más eficaz para promover el crecimiento económico y reducir el déficit fiscal. En este caso, los niveles de producción e inversión privada en el equilibrio final a largo plazo son mayores que en el equilibrio inicial, independientemente de que los choques sean previstos o no. Por otra parte, en el caso de la política de superávit primario, los niveles de producción e inversión privada en el equilibrio final a largo plazo son inferiores a los del equilibrio inicial, independientemente del tipo de choque.

\section{Estructura temporal de los tipos de interés}

Suponemos que los bonos perpetuos pagan un tipo de interés a largo plazo a través de un único cupón periódico con el valor fijado en una unidad monetaria determinada. Si $P(t)$ es el precio del bono y $R(t)$ es su tasa interna de retorno, tenemos que $R(t)=\frac{1}{P(t)}$.

En mercados eficientes y libres de riesgos, mediante el arbitraje, el tipo de interés a corto plazo $r(t)$ será igual al tipo de interés a largo plazo más el capital $\frac{\dot{P}(t)}{P(t)}$. Por lo tanto, $r(t)=\frac{1}{P(t)}+\frac{P(t)}{P(t)}$,
que origina la ecuación diferencial $\dot{P}(t)-r P(t)=-1$.

La solución de esta ecuación para $P(t)$ hacia el futuro, como se muestra en el anexo, da como resultado la siguiente expresión: $P(t)=\int_{t}^{\infty} e_{t}^{-\int_{t}^{s} r(w) d w} d s$. Siempre que $R(t)=\frac{1}{P(t)}$, el tipo de interés a largo plazo se puede definir como $R(t)=\frac{1}{\int_{t}^{\infty} e_{t}^{-\int_{t}^{s} r(w) d w} d s}$, definiendo $P(t, s)=e_{t}^{-\int_{t}^{s} r(w) d w \text { como el }}$ precio de un bono de cupón cero en un período $t$ que vence en la fecha $s$, por lo que tenemos que $P(t, s)=e_{t}^{-\int_{t}^{s} r(w) d w}$.

Sin embargo, según nuestra hipótesis, el bono a largo plazo tiene un valor nominal unitario, que deberá ser igual a la suma de los tipos a corto plazo considerando el precio para un período $t$ de un bono de cupón cero que vence en la fecha $s$. En otras palabras, $\int_{t}^{\infty} r(s) P(t, s) d s=1$.

Por consiguiente, el tipo de interés a largo plazo puede expresarse como:

$$
R(t)=\frac{\int_{t}^{\infty} r(s) P(t, s) d s}{\int_{t}^{\infty} P(t, s) d s}
$$


En otras palabras, el tipo de interés a largo plazo es un promedio ponderado de los tipos de interés a corto plazo perfectamente previstos para el futuro.

De las condiciones de equilibrio (7) y (8) obtenemos que $r=(1-\beta) f_{k}(K, l(\lambda, K) h[(1-\alpha) \bar{g}]$. Linealizando esta expresión alrededor del equilibrio de estado estacionario, encontramos que su trayectoria sigue la expresión: $r(t)=\tilde{r}+(1-\beta) w_{21}(k(t)-\widetilde{k})+(1-\beta) w_{22}(\lambda(t)-\tilde{\lambda})$, donde $\tilde{r}=\rho$. Por lo tanto, la dinámica de los tipos de interés a corto plazo se obtiene diferenciando la expresión $r(t)$ anterior 5 .

Por lo tanto:

$$
\dot{r}(t)=(1-\beta) w_{21} \dot{k}(t)+(1-\beta) w_{22} \dot{\lambda}(t)
$$

Como $R(t)$ es un promedio ponderado, el tipo de interés a corto plazo está perfectamente previsto para el futuro. Según (expresión 15), la dinámica se establece a partir del análisis de (16).

\section{Choques permanentes de $\alpha$ en los tipos de interés a corto y largo plazo}

Inicialmente, consideramos el caso de una política fiscal no prevista en la que la autoridad fiscal anuncia inmediatamente un aumento del gasto permanente en infraestructura (caída en $\alpha$ ). Según el análisis desarrollado en el punto 1 de la sección III, $\lambda(t)$ nmediatamente salta al brazo estable X'X'. Este crecimiento en $\lambda(t)$ provoca un aumento de la productividad marginal del capital que, al mismo tiempo, aumenta los tipos de interés a corto plazo. Por lo tanto, el tipo de interés a corto plazo también salta, seguido de una disminución gradual a lo largo del tiempo, como se muestra en el gráfico 3A.

\section{Gráfico 3}

\section{A. Choque no previsto}

$r(t), R(t)$

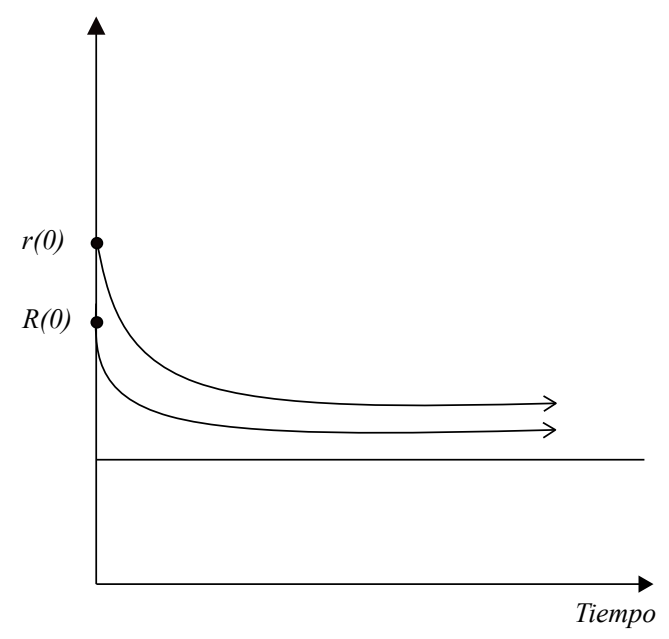

B. Choque previsto

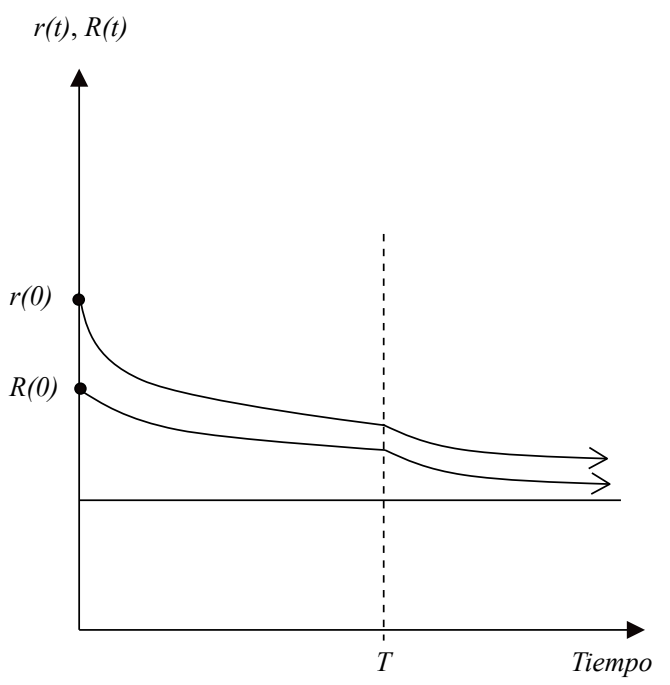

Fuente: Elaboración propia.

Dado que la dinámica de la tasa $r(t)$ se rige por la ecuación (16), y como después del șalto de $\lambda(t)$, $k(t)$, sigue una trayectoria creciente $(\dot{k}(t)>0)$ y $\lambda(t)$ sigue una trayectoria decreciente $\dot{\lambda}(t)<0)$, la tasa de interés a corto plazo disminuirá a lo largo del tiempo hasta alcanzar su equilibrio de estado estacionario $\rho$.

5 Buscando la dinámica de $R(t)$, siempre que $\dot{R}=R\left[R-(1-\beta) f_{k}(K, I(\lambda, K))\right]$, y linealizando esta expresión con el equilibrio de estado estacionario, obtenemos que: $\dot{R}(t)=\rho\left[(R(t)-\tilde{R})-(1-\beta) w_{21}(k(t)-\tilde{k})-(1-\beta) w_{22}(\lambda(t)-\tilde{\lambda})\right]$ en que $\dot{R}=\rho$. 
El tipo de interés a largo plazo se expresa como un promedio ponderado de los tipos de interés a corto plazo perfectamente previstos para el futuro según la expresión (15). El tipo de interés a largo plazo también salta de su equilibrio inicial $\rho$ para luego disminuir a través del tiempo, siempre retrasando la trayectoria de $r(t)$, hasta converger de nuevo a su nivel de equilibrio inicial. Como se muestra en la expresión (16), las convergencias de los tipos de interés a corto y largo plazo se ralentizan a medida que aumenta el superávit primario como porcentaje del PIB (aumento de $\beta$ ).

En el caso de un anuncio de aumento de los gastos de infraestructura en una fecha futura $T$ (política fiscal prevista) las trayectorias de convergencia de los tipos de interés son similares a las del caso anterior, pero con diferencias sutiles. Estas trayectorias se muestran en el gráfico 3B. En primer lugar, sus saltos iniciales son más limitados, ya que los agentes prevén que los cambios solo se producirán en un momento futuro $T$. En segundo lugar, tras el salto de $\lambda(t)$ a B en el gráfico $2 \mathrm{~B}, k(t)$ y $\lambda(t)$ siguen una trayectoria inestable decreciente hasta el brazo estable $X^{\prime} X^{\prime}$, cuando el cambio de política se produce en el momento $T$. De acuerdo con la expresión (16), en este mismo período de tiempo, la tasa de interés $r(t)$ disminuye, aunque en menor medida $\lambda(t)$ de lo que se observa en el gráfico 3B. Sobre la base del tiempo $T$, a medida que $k(t)$ crece y $\lambda(t)$ disminuye, el tipo de interés a corto plazo converge más rápidamente hacia su equilibrio inicial que en su trayectoria anterior.

\section{Choques permanentes en en $\beta$ los tipos de interés a corto y largo plazo}

Inicialmente, se considera el caso en que el gobierno anuncia un aumento inmediato del superávit primario como porcentaje del PIB (política fiscal no prevista). Como se ha visto antes, esto implica un aumento del parámetro $\beta$ de política fiscal.

En el gráfico 2C de la subsección III.2, un aumento $\beta$ provoca un pequeño salto en el tipo de interés a corto plazo en el momento en que se anuncia el cambio de política debido a la caída de la utilidad marginal de la riqueza. Después de la caída inicial de $\lambda(t)$, el acervo de capital $k(t)$ comienza a disminuir y $\lambda(t)$ crece. De acuerdo con la dinámica de la ecuación en (16), el tipo a corto plazo comienza a aumentar a través del tiempo hasta que vuelve a su equilibrio a largo plazo $\rho$, como se muestra en el gráfico 3C.

\section{Gráfico 3}

\section{Choque no previsto}

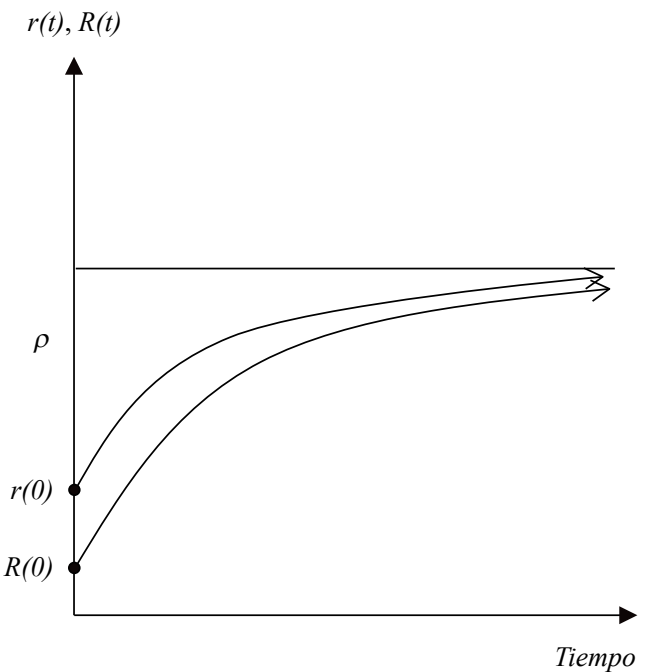

\section{Choque previsto}

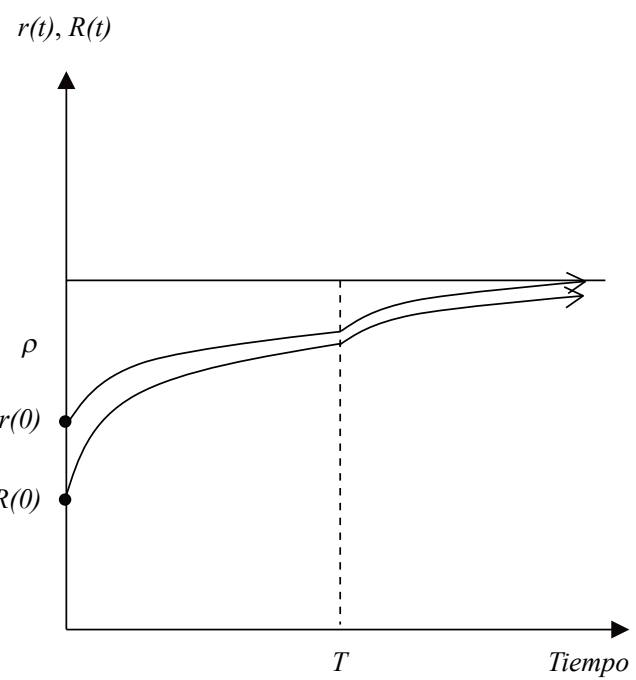

Fuente: Elaboración propia. 
Dado que el tipo de interés a largo plazo es un promedio ponderado de los tipos de interés a corto plazo perfectamente previstos para el futuro, también sufre una mayor caída inicial que en el caso de los tipos de interés a corto plazo. Después de esta caída inicial, refleja una trayectoria creciente, pero siempre por debajo del tipo de corto plazo, hasta que alcanza su nivel de equilibrio $\rho$ inicial. En el gráfico 3C se observa esta convergencia muy claramente.

Una vez más, es importante destacar que el aumento de la intensidad en $\beta$ retrasa aún más la convergencia de ambos tipos de interés.

Cuando el crecimiento del superávit primario se produce en una fecha futura $T$ (política fiscal prevista), los movimientos de los tipos de interés a corto y largo plazo no son muy diferentes. Lo mismo ocurre con la política fiscal no prevista: ambos tipos de interés disminuyen, pero en menor medida. En última instancia, los agentes descuentan el hecho de que solo habrá un cambio de política en una fecha futura (véase el gráfico 3D). A partir de estos saltos, ambos tipos de interés empiezan a crecer a través del tiempo, pero el tipo a largo plazo es siempre más bajo que el tipo a corto plazo. En el gráfico $2 \mathrm{D}$ se observa que después del salto inicial $\lambda(t)$, el acervo de capital y la utilidad marginal de la riqueza siguen trayectorias inestables (de $\mathrm{B}$ a $\mathrm{C}$ ) hasta que se alcanza $\mathrm{C}$, cuando en realidad hay un crecimiento del superávit primario. Esto sucede en un período de tiempo $T$. En estas trayectorias, a medida que $k(t)$ y $\lambda(t)$ decrecen a través del tiempo, podemos concluir de la ecuación (16) que el tipo de interés a corto plazo debe aumentar aunque la disminución en $\lambda(t)$ limita el crecimiento.

A partir del punto $\mathrm{C}, k(t)$ continúa su trayectoria descendente mientras que $\lambda(t)$ comienza a aumentar. El resultado es que el tipo de interés a corto plazo, después del período de tiempo $T$, sigue aumentando más rápidamente que en el período anterior. El tipo de interés a largo plazo refleja el mismo movimiento, pero su trayectoria hacia el equilibrio inicial siempre se mantiene más baja que la del tipo de interés a corto plazo. En última instancia, se trata simplemente de un promedio ponderado de los tipos a corto plazo perfectamente previstos por los agentes.

En este caso, la intensidad del parámetro de política fiscal $\beta$ frena la convergencia de ambos tipos de interés hacia su equilibrio final.

\section{Política fiscal y bienestar}

El análisis de los efectos de los cambios en los parámetros de la política fiscal sobre el bienestar considera la utilidad total de los agentes a una determinada tasa de descuento. Para este fin, se usa la trayectoria estable de utilidad instantánea linealizada alrededor de su estado estacionario 6 . Según Fisher y Turnovsky (1995) y Turnovsky (1995) la utilidad de los agentes $U\left(c(t), l(t), g_{c}(t)\right)$ se puede desglosar como: $U(t) \cong \widetilde{U}+(U(0)-\widetilde{U}) e^{\mu_{1} t}$, en que para simplificar adoptamos: $U(t)=U\left(c(t), l(t), g_{c}(t)\right)$.

Denota la utilidad total descontada que mide el bienestar del agente por $W, W=\int_{0}^{\infty}\left[\widetilde{U}+(U(0)-\widetilde{U}) e^{\mu_{1} t}\right] e^{-\rho t} d t$
$\widetilde{U}(U(0)-\widetilde{U})$ lo que implica que $W=\frac{\widetilde{U}}{\rho}+\frac{(U(0)-\widetilde{U})}{\rho-\mu_{1}}$.

En la expresión anterior, el término $\widetilde{U} / \rho$ representa el bienestar instantáneo descontado por el tipo intertemporal $\rho$. Este término expresa el nivel de bienestar resultante si se alcanza inmediatamente el equilibrio a largo plazo. El término $\frac{(U(0)-\widetilde{U})}{\rho-\mu_{1}}$ representa el ajuste hasta que se alcanza el equilibrio a largo plazo, ya que en una economía con acumulación de capital, el estado estable se alcanza gradualmente?

\footnotetext{
6 En este artículo no se analiza el resultado óptimo de las políticas presentadas. Véase un análisis detallado de métodos de optimización para políticas fiscales y monetarias en Chari y Kehoe (1999).

7 Véase una versión de tiempo discreto de ese enfoque en Stockman (2001).
} 
El objetivo principal de esta sección es encontrar una política presupuestaria que maximice el bienestar social. Específicamente, se procura obtener expresiones para $\widehat{\alpha}$ y $\widehat{\beta}$ de tal manera que:

$$
(\widehat{\alpha}, \widehat{\beta})=\underset{\alpha, \beta \in[0,1]}{\operatorname{argmax}} W
$$

Lamentablemente, no es posible obtener una solución general para (17) una vez que la dependencia de $U(0)$ y $\widetilde{U}$ en los parámetros $\alpha$ y $\beta$ está dada implícitamente por la función de utilidad del agente representativo y la tecnología de producción. Por lo tanto, en busca de una mejor solución al problema, destacaremos algunas especificaciones:

Inicialmente, el problema (17) puede ser sustituido por el problema equivalente:

$$
(\widehat{\alpha}, \widehat{\beta})=\underset{\alpha, \beta \in[0,1]}{\operatorname{argmax}} \bar{W}
$$

Donde $\bar{W}=\rho W$ en que $\bar{W}=\tau U(0)+(1-\tau) \widetilde{U}$ con $\tau=\rho /\left(\rho+\left|\mu_{1}\right|\right)$. Se supone que $\tau$ es independiente de $\alpha$ y $\beta$.

Además de los supuestos anteriores, se adoptarán formas funcionales para la utilidad de un consumidor representativo y para la tecnología de producción. Según Christiano, Eichenbaum y Rebelo (2011) se supone que la función de utilidad (instantánea) de un consumidor representativo es:

$$
U(c, l, \alpha \bar{g})=\frac{\left(c^{\gamma}(1-l)^{1-\gamma}\right)^{1-\sigma}-1}{1-\sigma}+\varphi \ln \alpha \bar{g} \text { se } \sigma \neq 1
$$

de modo que todos los parámetros de esta función de utilidad no son negativos con $0<\gamma<1$.

La función de producción, al mismo tiempo, sigue la sugerencia de Barro (1990) sobre el gasto público productivo, de tal manera que:

$$
F(k, l,(1-\alpha) \bar{g})=A k^{\delta} l^{1-\delta}((1-\alpha) \bar{g})^{\eta}
$$

donde todos los parámetros de la función especificada arriba son positivos y $0<\delta<1$. Obsérvese que el parámetro $\eta$ mide la elasticidad del producto con respecto a los gastos de infraestructura.

Por último, se asume que el régimen fiscal en $t=0$ y el del estado estacionario se tratarán como parámetros. Esto significa que si consideramos $y(t)$ como el producto per cápita de la economía, para cada política de austeridad $\beta$, tendremos que $\beta y(0)=T(0)-\bar{g}=$ so y $\beta \tilde{y}=\widetilde{T}-\bar{g}=\tilde{s}$.

Por lo tanto, el supuesto anterior significa que en el problema (18), $s_{0}$ y $\tilde{s}$ se tratarán como parámetros.

Con las especificaciones anteriores es posible obtener soluciones explícitas para las variables endógenas del modelo utilizando los datos iniciales, así como para el estado estacionario. Según la proposición 1 del anexo, las soluciones para la oferta de mano de obra y el consumo privado para $t=0$ son respectivamente: a) $l(0)=\left[\frac{s_{0}}{\beta A k_{0}^{\delta}((1-\alpha) \bar{g})^{\eta}}\right]^{\frac{1}{1-\delta}}$ y b) $c(0)=\frac{\gamma(1-\delta) s_{0}(1-l(0)}{\beta(1-\gamma) l(0)}$.

En el estado estacionario, las soluciones para el consumo, la oferta de mano de obra y el acervo de capital son iguales a: c) $\tilde{l}=\frac{(1-\gamma)(\tilde{s}-\beta \bar{g})}{(1-\gamma)(\tilde{s}-\beta \bar{g})+\gamma(1-\delta)(1-\beta) \tilde{s}}$, d) $\tilde{c}=\frac{\tilde{s}-\beta \bar{g}}{\beta}$ y e) $\tilde{k}=\frac{(1-\beta) \tilde{\delta} s}{\rho \beta}$.

Sustituyendo estas soluciones ( $a, b, c, d$ y e) en (19), después de algunas manipulaciones algebraicas, obtenemos las siguientes expresiones para $U(0)$ y $\widetilde{U}$ :

$$
U(0)=\left[\frac{\gamma(1-\delta)}{\beta(1-\gamma)}\right] \frac{s^{\frac{-\gamma \delta(1-\delta)}{1-\delta}}\left[\phi(\alpha)^{\frac{1}{1-\delta}}-s_{0}^{\frac{1}{1-\delta}}\right]^{1-\delta}}{\phi(\alpha)^{\frac{(1-\gamma)(1-\sigma)}{1-\sigma}}}+\varphi \ln \alpha \bar{g}-\frac{1}{1-\sigma}
$$




$$
\widetilde{U}=\frac{(1-\gamma)^{(1-\gamma)(1-\sigma)}(\tilde{s}-\beta \bar{g})^{(1-\sigma)}}{\beta^{\gamma(1-\delta)}(1-\delta)[(1-\gamma)(\tilde{s}-\beta \bar{g})+\gamma(1-\delta)(1-\beta) \bar{s}]}+\varphi \ln \alpha \bar{g}-\frac{1}{1-\sigma}
$$

donde $\phi(\alpha)=\beta A k_{0}^{\delta}((1-\alpha) \bar{g})^{\eta}$

Antes de investigar las condiciones de primer orden para este problema (18), es importante verificar si existen soluciones de esquina, ya que deben limitarse al intervalo unitario. Inicialmente, las soluciones $\beta=0$ y $\alpha=1$ pueden descartarse a medida que la función objetiva se interrumpe, por lo que el uso de las condiciones de primer orden es imposible. También podemos observar que la función objetivo depende de $\alpha$, que tiende a ser más finita cuando $\alpha$ está cerca de cero. Por lo tanto, la solución $\alpha=0$ no puede ser óptima.

No se puede descartar la solución $\beta=1$ inmediatamente. Sin embargo, si así fuera, la economía desarrollaría una trayectoria en la que se destruirían los activos, ya que no habría acumulación. En consecuencia, el problema se tratará como un problema de maximización sin restricciones y se analizarán las condiciones de primer orden si $\beta=1$ cumple estas condiciones.

Utilizando las ecuaciones (20) y (21), las condiciones de primer orden en (18) para $\alpha$ y $\beta$ están dadas respectivamente por 8 :

$$
\begin{aligned}
& \left.\left[\frac{\tau \eta(1-\sigma)}{1-\sigma}\right] \frac{\gamma(1-\delta) s_{0}}{\widehat{\beta}(1-\gamma)}\right]^{\gamma(1-\sigma)} \frac{\left[\phi(\widehat{\alpha})^{\frac{1}{1-\sigma}}-s_{0}^{\frac{1}{1-\delta}}\right]^{-\sigma}\left[\gamma \phi(\widehat{\alpha})^{\frac{1}{1-\delta}}+(1-\gamma) s_{0}^{\frac{1}{1-\delta}}\right.}{\phi(\widehat{\alpha})^{\frac{(1-\gamma)(1-\sigma)}{1-\delta}}}=\frac{1-\widehat{\alpha}}{\widehat{\alpha}} \\
& x\left\{-\gamma(1-\sigma) \widehat{\beta}^{-1}(\tilde{s}-\widehat{\beta} \bar{g})+\frac{[(1-\gamma) \delta \sigma \tilde{s}-\gamma(1-\sigma) \bar{g}](\tilde{s}-\widehat{\beta} \bar{g})-\delta \sigma(1-\widehat{\beta}) \bar{g}}{(1-\sigma)(\tilde{s}-\widehat{\beta} \bar{g})+\delta \sigma(1-\widehat{\beta}) \tilde{s}}\right\}=0
\end{aligned}
$$

Obsérvese que una solución explícita para (22) y (23) solo es posible en casos muy especiales (algunos de los cuales se analizarán más adelante). Sin embargo, es posible llegar a algunas conclusiones cualitativas sobre las normas óptimas de austeridad y la composición del presupuesto sin necesidad de soluciones explícitas.

\footnotetext{
8 Los detalles del proceso se encuentran en el anexo.
} 
Utilizando la ecuación (22), la proposición 2 del anexo afirma que si $\eta>\left[\left(\frac{1-\gamma}{\gamma}\right)^{\gamma(1-\sigma)}\right] / \tau(1-\sigma)$, entonces cualquier solución válida para $\alpha$ debe ser tal que $\alpha<0,5$. Esto lleva a la siguiente conclusión: si la elasticidad de la producción con respecto al gasto en infraestructura es suficientemente alta y si el volumen de empleo en la fecha inicial representa más de la mitad de la fuerza de trabajo disponible, la solución socialmente óptima prescribe que la mayor parte del presupuesto público debe asignarse a inversiones (gasto en infraestructura). Para tener una idea de la magnitud de $\eta$ necesaria para validar la proposición 2, consideremos un ejemplo numérico. Supongamos que el ocio y el consumo se ponderan por igual en la utilidad del agente representativo y que la elasticidad de la sustitución intertemporal es igual a 2. En este caso, tenemos que $\gamma=0,5$ y $\sigma=0$ de modo tal que la condición para la elasticidad de la producción con respecto al gasto público en infraestructura es $\eta>1 / \tau$. Esto implica que cuanto menor sea la importancia de la utilidad hoy en día en una relación a largo plazo, mayor será el nivel $\eta$ necesario para tener como óptimo social que $\alpha<0,5$.

El resultado anterior merece cierta consideración. La relación inversa entre la elasticidad de la producción y el bienestar a corto plazo significa que, en primer lugar, $\eta>1$, lo que significa que para garantizar una condición social óptima, es necesario destinar la mayor parte del presupuesto a la inversión. La producción económica debe ser elástica en lo que respecta a la inversión, porque, como se especifica en la función de producción de Cobb-Douglas, tanto el capital privado como el público representan algún nivel de sustitución ${ }^{9}$, de modo que cuanto mayor sea el valor $\eta$, menor será la sustitución. Por consiguiente, en la composición de la producción económica, el capital público y el privado se complementarán mutuamente.

Los argumentos anteriores se suman al análisis de los choques fiscales en el equilibrio a largo plazo. Como se ha visto en la sección III, un aumento del porcentaje de gasto asignado a la infraestructura siempre aumenta el acervo de capital en un estado estacionario. Por lo tanto, considerando los efectos a largo plazo, cuanto más alto sea el valor de $\eta$, más fuerte será el impacto en la producción resultante de un aumento en el gasto de infraestructura, ya que tanto el capital privado como el público crecerán de forma complementaria.

Por último, un valor más bajo $\tau$ significa un $\eta$ más alto, lo que implica que la importancia a largo plazo en la composición del bienestar aumentará, reforzando así los argumentos a favor de que el capital privado y el público se complementen más.

Obsérvese que el resultado $\alpha<0,5$ es independiente del valor de $\beta$ en la situación socialmente óptima. Por lo tanto, en este caso la expresión $\widetilde{\beta}=\frac{\widetilde{T}-\widetilde{g}}{\tilde{y}}=\frac{\tilde{s}}{\widetilde{y}}$ es suficiente para determinar la regla de austeridad fiscal óptima, donde $\tilde{y}$ es la producción en el estado estacionario que se obtiene al aplicar la proposición 1.

Obsérvese que, aunque la solución propuesta para $\widetilde{\beta}$ es implícita, su escala depende claramente de la magnitud del superávit primario (carga fiscal) deseado por la autoridad fiscal y del tamaño del producto en equilibrio a largo plazo. Concretamente, mediante una diferenciación implícita, se puede demostrar que el signo de $d \widetilde{\beta} / d s$ depende de $1-\varepsilon_{y, s}$, donde $\varepsilon_{y, s}$ es la elasticidad del producto en relación con la magnitud del superávit primario determinado por la autoridad fiscal (ambos en condiciones de largo plazo). Como esta elasticidad es siempre positiva (por definición) el signo de $d \widetilde{\beta} / d s$ depende de la magnitud de $\varepsilon_{y, s}$.

Más específicamente, si el producto es inelástico con respecto al superávit primario, entonces cuanto mayor sea el superávit primario, mayor será la magnitud de $\widehat{\beta}$. Este es un resultado intuitivo y, a primera vista, es el único posible. Sin embargo, el modelo crea una posibilidad de que un aumento

9 Concretamente, la tasa marginal de sustitución técnica entre el capital privado y el público viene dada por $\frac{\delta(1-\alpha) \bar{g}}{\eta k}$. 
de $s$ que pueda verse acompañado por una reducción en $\beta$. Para ello, basta con que la producción económica sea elástica en relación con el superávit primario, lo que significa que si $\varepsilon_{y, s}>1$ el signo del derivado $d \widetilde{\beta} / d s$ es negativo.

¿Cuál es la razón de este resultado? Inicialmente, parece razonable suponer que la magnitud de $\varepsilon_{y, s}$ depende de la influencia del gobierno en la economía (medida por la relación $\bar{g} / y$ ) de la siguiente manera: cuanto mayor sea la influencia del gobierno en la economía, más intenso será el impacto de $\bar{g} / y$ lo suficientemente grande, ocurre que $\varepsilon_{y, s}>1$. Naturalmente, esta conjetura no ha sido probada a través de una proposición general o una regularidad empírica. En cualquier caso, ambos análisis están fuera del alcance de esta investigación ${ }^{10}$.

\section{Conclusiones}

En el caso de la política fiscal no prevista, el anuncio de un aumento inmediato y permanente de los gastos de infraestructura provoca una caída (salto) a corto plazo de la riqueza privada, lo que da lugar a una disminución del consumo y un crecimiento de la oferta de mano de obra. A partir de ese momento, se inicia una dinámica de equilibrio inicial para un nuevo equilibrio a largo plazo (equilibrio de estado estacionario). Al igual que en el momento inicial, se produce una caída en el consumo privado y el acervo de capital comienza a aumentar hacia un nuevo equilibrio a largo plazo. Simultáneamente, la utilidad marginal de la riqueza comienza a disminuir después de su crecimiento inicial. Sin embargo, el tiempo que la utilidad marginal de la riqueza, el consumo privado y la oferta de mano de obra tarda en alcanzar un nuevo equilibrio de estado estacionario es más largo, ya que el superávit primario crece como porcentaje del PIB.

En el equilibrio final a largo plazo, el consumo privado, el acervo de capital (inversión), la producción y la oferta de mano de obra son mayores que en el estado de equilibrio inicial.

En el caso de la política fiscal prevista, los efectos del aumento de los gastos de infraestructura en la producción, el consumo y el acervo de capital son similares a los que se observan en el caso de la política fiscal no prevista. La principal diferencia es el salto más limitado de la utilidad marginal de la riqueza a corto plazo.

Cabe señalar también que los efectos de un aumento del gasto de consumo del gobierno en la producción, el consumo privado, la inversión y la oferta de mano de obra son simétricos a los que se producen en el caso de un aumento de los gastos de infraestructura, independientemente de que el anuncio sea previsto o no.

En el caso de la política fiscal no prevista relacionada con el aumento del superávit primario a corto plazo, el consumo mejora y la oferta de mano de obra disminuye, lo que da lugar a una disminución de la productividad marginal de la mano de obra. A largo plazo, la producción, el consumo privado y el acervo de capital son menores y la oferta de mano de obra crece en comparación con el equilibrio inicial.

En resumen, concluimos que, en términos de generación de crecimiento económico, la política fiscal de aumento del gasto público en infraestructura es más eficaz que la política de mantenimiento de un superávit primario como proporción del PIB. Por lo tanto, esta política de gasto en infraestructura combinada con la política de gasto real constante que aborda el déficit fiscal son más eficaces para promover el crecimiento económico.

\footnotetext{
${ }^{10}$ El punto puede introducirse de manera menos drástica si se asume que la relación entre las magnitudes $\varepsilon_{y, s}$ y $\bar{g} / y$ es probabilística. Específicamente, usando la notación $G=\bar{g} / y$, puede aceptarse que $\operatorname{Pr}\left[\varepsilon_{y, s}>\frac{1}{G}\right]$ es una función $\mathrm{G}$ creciente.
} 
En cuanto a la estructura temporal de los tipos de interés, la política fiscal no prevista de aumento de los gastos de infraestructura provoca un salto inmediato del tipo de interés a corto plazo, seguido de una trayectoria decreciente hacia el equilibrio a largo plazo. Dado que el tipo de interés a largo plazo es un promedio ponderado de los tipos de interés a corto plazo perfectamente previstos para el futuro, también salta, pero en menor medida, antes de converger rápidamente hacia un equilibrio de estado estacionario. Sin embargo, cuanto más alto sea el objetivo de superávit primario, más tiempo tardarán ambos tipos de interés en alcanzar sus nuevos equilibrios.

En el caso de la política fiscal prevista, en la que el aumento del superávit primario tendrá lugar en una fecha futura, los movimientos a corto y largo plazo son similares a la situación descrita anteriormente. Esto ocurre también en el caso de la política no prevista, ya que ambos tipos de interés también disminuyen, pero lo hacen de una manera menos drástica. Al final, los agentes descuentan el hecho de que solo habrá un cambio de política en el futuro. Después de caer, ambos tipos de interés empiezan a subir de nuevo a través del tiempo, pero con la trayectoria del tipo a largo plazo siempre por detrás de la del tipo a corto plazo. Desde el momento en que se produce el cambio de política, ambos tipos empiezan a aumentar más rápidamente en comparación con el período anterior.

En este caso, la magnitud del superávit primario frena la convergencia de ambos tipos de interés hacia su equilibrio final a largo plazo.

Por último, los resultados de las políticas fiscales y presupuestarias que maximizan el bienestar social sugieren que si la producción es suficientemente sensible al gasto en infraestructura o si el corto plazo no es muy relevante en la utilidad total, ajustada a una tasa de descuento, dada la mayor parte de los recursos públicos deben asignarse al gasto en inversión, siempre que el total de trabajadores empleados sea superior al $50 \%$ de la fuerza de trabajo disponible. El porcentaje del PIB asignado al superávit primario depende de la elasticidad del ingreso per cápita con respecto al desempeño fiscal de la economía. Concretamente, si el ingreso per cápita es elástico con respecto al superávit primario, incluso si la autoridad fiscal decide un aumento absoluto de este superávit, el porcentaje asignado del PIB puede terminar siendo inferior. 


\section{Bibliografía}

Agénor, P. (2005), "Fiscal adjustment and labor market dynamics in an open economy", Journal of Development Economics, vol. 76, № 1, febrero.

Aschauer, D. (2008), “¿Es productivo el gasto público?”, Papeles de Economía Española, №118. (1990), "Why is infrastructure important?", Is There a Shortfall in Public Capital Investment?, A. Munnell (ed.), Conference Series, №34, Boston, Banco de la Reserva Federal de Boston.

(1988), "The equilibrium approach to fiscal policy", Journal of Money, Credit and Banking, vol. 20, $N^{\circ} 1$, febrero.

Barro, R. (1990), "Government spending in a simple model of endogenous growth", Journal of Political Economy, vol. 98, N 5.

Baxter, M. y R. King (1993), "Fiscal policy in general equilibrium", The American Economic Review, vol. 83, $N^{\circ} 3$, junio.

Blanchard, O. (1981), "Output, the stock market, and interest rates", The American Economic Review, vol. 71, No 1, marzo.

Chari, V. y P. Kehoe (1999), "Optimal fiscal and monetary policy", NBER Working Paper, № 6891, Cambridge, Oficina Nacional de Investigaciones Económicas (NBER), enero.

Christiano, L., M. Eichenbaum y S. Rebelo (2011), "When is the government spending multiplier large?", Journal of Political Economy, vol. 119, № 1, febrero.

Cox, J., J. Ingersoll y S. Ross (1985), "A theory of the term structure of interest rates", Econometrica, vol. 53, $\mathrm{N}^{\circ}$ 2, marzo.

De la Fuente, A. (2000), Mathematical Methods and Models for Economists, Nueva York, Cambridge University Press.

Fisher, W. y S. Turnovsky (1995), "The composition of government expenditure and its consequences for macroeconomic performance", Journal of Economic Dynamics and Control, vol. 19, Nㅜ 4, mayo.

_ (1992), "Fiscal policy and the term structure of interest rates: an intertemporal optimizing analysis", Journal of Money, Credit and Banking, vol. 24, № 1, febrero.

Hirsch, M. y S. Smale (1983), Ecuaciones diferenciales, sistemas dinámicos y álgebra lineal, Madrid, Alianza Editorial.

IPEA (Instituto de Investigación Económica Aplicada) (2016), "Ipeadata” [en línea] http://www.ipeadata.gov.br.

Marinho, E. y M. Benegas (2006), "Ajuste fiscal no Brasil: por que adotar o regime de superávit primário ou o de déficit nominal zero?" [en línea] http://www.tesouro.fazenda.gov.br/premio/Premio_TN/XIpremio/ divida/1afdpXIPTN/1premio_afdp.pdf.

McCafferty, S. (1986), "Aggregate demand and interest rates: a macroeconomic approach to the term structure", Economic Inquiry, vol. 24, № 4.

Stockman, D. (2001), "Balanced-budget rules: welfare loss and optimal policies", Review of Economic Dynamics, vol. 4, № 2, abril.

Turnovsky, S. (1995), Methods of Macroeconomic Dynamics, Cambridge, MIT Press.

Turnovsky, S. y M. Miller (1984), "The effects of government expenditure on the term structure of interest rates", Journal of Money, Credit and Banking, vol. 16, № 1, febrero.

Woodford, M. (1996), "Control of the public debt: a requirement for price stability?", NBER Working Paper, №5684, Cambridge, Oficina Nacional de Investigaciones Económicas (NBER), julio. 


\section{Anexo A1}

\section{Estática comparativa a corto plazo}

Las ecuaciones (5) y (6) de la sección II ayudan a encontrar soluciones para el consumo $c(t)$ y la oferta de mano de obra $l(t)$ como funciones de la utilidad marginal de la riqueza $\lambda(t)$, del acervo de capital $k(t)$ y de los parámetros de política $\alpha$ y $\beta$. Por lo tanto, diferenciando (5) y (6), encontramos que:

$$
\begin{gathered}
U_{c c} d c+U_{c l} d l=d k \\
U_{l c} d c+\left(U_{l l}+\lambda(1-\beta) f_{l l} h\right) d l=-\lambda(1-\beta) f_{k l} d k-(1-\beta) f h d \lambda+\lambda(1-\beta) h^{\prime} \bar{g} d \alpha+\lambda f h d \beta
\end{gathered}
$$

La solución del sistema anterior origina las siguientes relaciones a corto plazo, tal como se presentaron en el punto 1 de la sección II:

$$
\begin{gathered}
\frac{d c}{d k}=\frac{\lambda(1-\beta) f_{k} U_{c l} h}{\Delta}<0, \frac{d l}{d k}=-\frac{\lambda(1-\beta) f_{k} U_{c c} h}{\Delta}>0, \frac{d c}{d l}=\frac{U_{l l}+\lambda(1-\beta) f_{l l} h+(1-\beta) f_{i} h}{\Delta}<0, \\
\frac{d l}{d \lambda}=-\frac{(1-\beta) f_{i} U_{c c} h+U_{c}}{\Delta}>0, \frac{d c}{d \alpha}=-\frac{\lambda(1-\beta) f_{i} U_{c l} h \bar{g}}{\Delta}>0, \frac{d c}{d \beta}=-\frac{\lambda f U_{c l} h}{\Delta}>0 \text { y } \frac{d l}{d \beta}=\frac{\lambda f U_{c c} h}{\Delta}<0
\end{gathered}
$$

\section{Demostración de que el equilibrio a largo plazo es un punto de silla}

El sistema de ecuaciones linealizadas (13) y (14) alrededor del estado estacionario puede escribirse en forma de matriz como:

$$
\begin{gathered}
\dot{k}=w_{11}(k-\tilde{k})+w_{12}(\lambda-\tilde{\lambda}) \\
\dot{\lambda}=-(1-\beta) \tilde{\lambda} w_{12}(k-\widetilde{k})-(1-\beta) \tilde{\lambda} w_{22}(\lambda-\tilde{\lambda})
\end{gathered}
$$

Para demostrar que el equilibrio del sistema es un tipo de punto de silla, todavía tenemos que probar que el determinante del sistema anterior es negativo. De hecho, el signo determinante es $-\tilde{\lambda}(1-\beta)\left[w_{11} w_{22}-w_{21} w_{12}\right]<0, w_{11}>0, w_{22}>0, w_{12}>0, w_{21}<0$ y $0<\beta<1$. Además, como $-\tilde{\lambda}(1-\beta)\left[w_{11} w_{22}-w_{12} w_{21}\right]=\mu_{1} \mu_{2}$ donde $\mu_{1}$ y $\mu_{2}$ son los valores propios asociados con la matriz del sistema mencionado arriba, admitiremos sin pérdida de generalidad que $\mu_{1}<0$ y $\mu_{2}>0$.

\section{Brazos estables e inestables de equilibrio del punto de silla, determinación de trayectorias de $k(t)$ y $\lambda(t)$ y salto $(t)$}

Se acepta que el gobierno puede anunciar hoy un cambio en la política fiscal en un futuro $T>0$. En otras palabras, el cambio de política es previsto por los agentes. Inicialmente, la solución para $k(t)$ y $\lambda(t)$ en el período $0 \leq t<T$ es la siguiente:

$$
\begin{gathered}
k(t)=\widetilde{k}_{1}+A_{1} e^{\mu_{1} t}+A_{2} e^{\mu_{2} t} \\
\lambda(t)=\widetilde{\lambda}_{1}+A_{1}\left(\frac{\mu_{1}-w_{11}}{w_{12}}\right) e^{\mu_{1} t}+A_{2}\left(\frac{\mu_{2}-w_{11}}{w_{12}}\right) e^{\mu_{2} t}
\end{gathered}
$$

Para el período $t \geq T$, las soluciones para $k(t)$ y $\lambda(t)$ son: 


$$
\begin{gathered}
k(t)=\widetilde{k} 2+A_{1}^{\prime} e^{\mu_{1} t}+A_{2}^{\prime} e^{\mu_{2} t} \\
\lambda(t)=\widetilde{\lambda}_{2}+A_{1}^{\prime}\left(\frac{\mu_{1}-w_{11}}{w_{12}}\right) e^{\mu_{1} t}+A_{2}^{\prime}\left(\frac{\mu_{2}-w_{11}}{w_{12}}\right) e^{\mu_{2} t}
\end{gathered}
$$

Para cumplir con la condición de transversalidad (9), consideramos que $A_{2}{ }_{2}=0$. Imponiendo esta restricción a las dos ecuaciones anteriores, tenemos el brazo estable del equilibrio del punto de silla:

$$
\lambda(t)=\tilde{\lambda}_{2}+\left(\frac{\mu_{1}-w_{11}}{w_{12}}\right)(k(t)-\widetilde{K})
$$

donde el coeficiente angular de esta línea recta es negativo, ya que $w_{11}>0, w_{12}>0, \mu_{1}<0$.

El brazo inestable se obtiene sustituyendo la raíz estable $\mu_{1}$ por la raíz inestable $\mu_{2}$ en la anterior ecuación en línea recta. Por lo tanto:

$$
\lambda(t)=\widetilde{\lambda}_{2}+\left(\frac{\mu_{2}-w_{11}}{w_{12}}\right)(k(t)-\widetilde{K})
$$

El coeficiente angular de la línea de brazo inestable es positivo, ya que según las propiedades de los valores propios considerando que $\left(\frac{\mu_{2}-w_{11}}{w_{12}}\right)=\left(\frac{-\tilde{\lambda}(1-\beta) w_{21}}{\mu_{2}+\tilde{\lambda}(1-\beta) w_{22}}\right)$, considerando que $w_{21}<0$,
$w_{22}>0$ y que $\mu_{2}>0$.

Con el fin de obtener las trayectorias de $k(t)$ y $\lambda(t)$, tenemos que determinar las constantes $A_{1}, A_{2}$ y $A_{1}^{\prime}$ ya que $A_{2}^{\prime}=0$. Para ello hemos desarrollado dos hipótesis adicionales: la primera es que el acervo de capital inicial (fecha $t=0$ ) es igual al acervo de capital inicial de estado estacionario, que es $k(0)=\widetilde{k} 1$; la segunda es que en la fecha del cambio de política $(t=T)$ hay una continuidad de soluciones, lo que significa que deben ser similares para este período de tiempo.

Por lo tanto, sobre la base de la primera hipótesis, $k(0)=\widetilde{k}_{1}+A_{1}+A_{2}$ lo que implica que que $A_{1}+A_{2}=0 \circ A_{1}=-A_{2}$. La segunda hipótesis origina el siguiente sistema de ecuaciones:

$$
\begin{gathered}
\left(A_{1}^{\prime}-A_{1}\right)\left(\frac{\mu_{1}-w_{11}}{w_{12}}\right) e^{\mu_{1} t}+A_{2}\left(\frac{\mu_{2}-w_{11}}{w_{12}}\right) e^{\mu_{2} T}=\widetilde{\lambda}_{2}-\tilde{\lambda}_{1} \\
\left(A_{1}^{\prime}-A_{1}\right) e^{\mu_{1} T}+A_{2} e^{\mu_{2} T}=\widetilde{k}_{2}-\widetilde{k}_{2}
\end{gathered}
$$

Por lo tanto, la solución del sistema anterior, además de utilizar la restricción $A_{1}=-A_{2}$ produce las siguientes soluciones:

$$
\begin{gathered}
A_{1}=\frac{e^{\mu_{1} T}\left[\left(\frac{\mu_{1}-w_{11}}{w_{12}}\right)\left(\widetilde{k}_{2}-\widetilde{k}_{2}\right)-\left(\tilde{\lambda}_{2}-\widetilde{\lambda}_{1}\right)\right]}{\Delta} \\
A_{1}^{\prime}=\frac{e^{\mu_{1} T}\left[\left(\frac{\mu_{1}-w_{11}}{w_{12}}\right)\left(\widetilde{k}_{2}-\widetilde{k}_{2}\right)-\left(\widetilde{\lambda}_{2}-\widetilde{\lambda}_{1}\right)\right]+e^{\mu_{2} T}\left[\left(\widetilde{\lambda}_{2}-\widetilde{\lambda}_{1}\right)-\left(\frac{\mu_{2}-w_{11}}{w_{12}}\right)\left(\widetilde{k}_{2}-\widetilde{k}_{1}\right)\right.}{\Delta}
\end{gathered}
$$

donde $\Delta=e^{\left(\mu_{1}+\mu_{2}\right) T}\left(\frac{\mu_{2}-\mu_{1}}{w_{12}}\right)>0$.

Así, sustituyendo $A_{1}, A_{2}$ y $A^{\prime}{ }_{1}$ en las expresiones $k(t)$ y $\lambda(t)$ para cada uno de los períodos $0 \leq t<T$ y $t \geq T$, determinamos plenamente las trayectorias de $k(t)$ y $\lambda(t)$. Para determinar la solución de este tipo de modelo, se admite generalmente que una de las variables puede saltar con cada nueva medida anunciada por el gobierno mientras que la otra evolucionará continuamente a través del tiempo. Para este artículo, la primera variable será la utilidad marginal de la riqueza $\lambda(t)$. La variable $k(t)$ será la variable predeterminada que evoluciona continuamente a través del tiempo.

Para calcular la dimensión de este salto, es suficiente tener $t=0$ en la ecuación de $\lambda(t)$ para un período $0 \leq t<T$ observando que $A_{1}=-A_{2}$. Por lo tanto, concluimos que:

$$
\lambda(0)=\tilde{\lambda}_{1}+A_{2}\left(\frac{\mu_{2}-\mu_{1}}{w_{12}}\right)
$$


Reemplazando el valor de $A_{2}$ en esta última expresión, obtenemos la expresión para el salto $\lambda(t)$ :

$$
\lambda(0)-\widetilde{\lambda}_{1}=e^{-\mu_{2} T}\left[\left(\tilde{\lambda}_{2}-\tilde{\lambda}_{1}\right)-\left(\frac{\mu_{1}-w_{11}}{w_{12}}\right)\left(\tilde{k}_{2}-\widetilde{k}_{1}\right)\right]
$$

Independientemente del signo de la expresión entre corchetes en la ecuación anterior, cuanto más largo sea el tiempo $T$, es decir, cuanto más lejano sea el cambio de política anunciado, menor será el salto $\lambda(t)$ resultante.

Cuando el cambio de política se produce inmediatamente (hoy), lo que significa que no es previsto por los agentes, el salto $\lambda(t)$ se calcula haciendo que $T=0$. Por lo tanto, tenemos que:

$$
\lambda(0)=\tilde{\lambda}_{2}-\left(\frac{\mu_{1}-w_{1}}{w_{12}}\right)\left(\widetilde{k}_{2}-\widetilde{k}_{1}\right)
$$

que es solo la expresión del brazo estable. Esto implica que cuando el anuncio de la política no se prevé, $\lambda(t)$ inmediatamente salta a la línea recta del equilibrio del punto de silla del brazo estable.

La característica esencial de las soluciones en este tipo de modelo es que la dinámica implica tres fases. Un anuncio hoy sobre una alteración de la política en un tiempo futuro $T$ genera un salto inmediato en $\lambda(t)$. Cuanto más lejos en el futuro esté el cambio de política, más débil es la intensidad del salto. Inmediatamente después, estas variables siguen trayectorias inestables hasta alcanzar el equilibrio del punto de silla del brazo estable en un período $T$. A partir de este punto, estas variables continúan en el brazo estable hasta alcanzar el nuevo equilibrio de estado estacionario.

Cuando el anuncio del cambio de política y su aplicación son inmediatos, la utilidad marginal de la riqueza salta inmediatamente al brazo estable y es seguida por $k(t)$ y $\lambda(t)$ en su trayectoria a través del brazo estable hacia el nuevo equilibrio del estado estacionario.

\section{Solución formal de la ecuación $\dot{P}(t)-r(t) P(t)=-1$ hacia adelante}

La solución general de esta ecuación diferencial viene dada por la siguiente expresión:

$$
P(t)=e_{0}^{s} r(t) d t A-\int_{0}^{t} e^{-\int_{0}^{t} r(s) d s} d s
$$

donde $A$ es una constante a ser determinada. Para asegurar que esta constante se establezca de forma endógena, Sargent y Wallace (1973) propusieron la aplicación de la condición terminal en lugar de la condición inicial. La condición terminal es que el nivel de precios debe permanecer limitado cuando $t \rightarrow \infty$. Para este propósito, debemos tener:

$$
A=\lim _{t \rightarrow \infty} \int_{0}^{t} e^{-\int_{0}^{t} r(s) d s} d s=\int_{0}^{\infty} e^{-\int_{0}^{t} r(s) d s} d s
$$

Reemplazando este último resultado en la expresión $P(t)$, encontramos que:

$$
P(t)=e_{0}^{\int_{0}^{s} r(t) d t}\left[\int_{t}^{\infty} e_{0}^{-\int_{0}^{t} r(w) d w} d s\right]=\int_{t}^{\infty} e_{t}^{-\int_{t}^{s} r(w) d w} d s
$$

Siempre que $(t)=\frac{1}{P(t)^{\prime}}$, finalmente encontramos que:

$$
R(t)=\frac{1}{\int_{t}^{\infty} e_{t}^{-\int_{t}^{s} r(w) d w} d s}
$$




\section{Demostración de la proposición 1}

Proposición 1: Supongamos que se cumplen las condiciones (17) - (19). Entonces:
a) $l(0)=\left[\frac{s_{0}}{\beta A k_{0}^{\delta}((1-\alpha) \bar{g})^{\eta}}\right]^{\frac{1}{1-\delta}}$;
b) $c(0)=\frac{\gamma(1-\delta) s o(1-l(0))}{\beta(1-\gamma) l(0)}$;
c) $\tilde{c}=\frac{\tilde{s}-\beta \bar{g}}{\beta}$;
d) $\tilde{l}=\frac{(1-\gamma)(\tilde{s}-\beta \bar{g})}{(1-\gamma)(\tilde{s}-\beta \bar{g})+\gamma(1-\delta)(1-\beta) \tilde{s}}$;
e) $\tilde{k}=\frac{(1-\beta) \delta \tilde{s}}{\rho \beta}$

\section{Demostración:}

a) En $t=0$, tenemos que:

$$
\beta A k_{0}^{\delta} I(0)^{1-\delta}((1-\alpha) \bar{g})^{\eta}=s_{0}
$$

Resolviendo A.1 para l(0), obtenemos el resultado deseado.

b) Utilizando las funciones de utilidad y utilidad tecnológica, las ecuaciones (5) y (6) pueden escribirse:

$$
\begin{gathered}
\gamma c^{\gamma(1-\sigma)-1}(1-l)^{(1-\gamma)(1-\sigma)}=\lambda \\
(1-\gamma) c^{\gamma(1-\sigma)}(1-l)^{(1-\gamma)(1-\sigma)-1}=\lambda(1-\delta) A k^{\delta} l^{(1-\delta)-1}((1-\alpha) \bar{g})^{\eta}
\end{gathered}
$$

Combinando (A.2) y (A.3) en $t=0$ y usando la definición de $y$ tenemos que:

$$
\left(\frac{1-\gamma}{\gamma}\right) \frac{l(0) c(0)}{(1-l(0))}=(1-\delta) y(0)
$$

El resultado se demuestra considerando el supuesto de que $y(0)=s_{0} / \beta$ y el ítem a) de la ecuación (A.4).

En esta situación, las condiciones de primer orden (5) y (6) junto con las ecuaciones que describen el equilibrio en estado estacionario producen el siguiente sistema:

$$
\begin{gathered}
(1-\beta) \tilde{y}=\tilde{c}+\bar{g} \\
(1-\beta) \delta \tilde{y}=\rho \tilde{k} \\
\left(\frac{1-\gamma}{\gamma}\right) \frac{\tilde{l} \tilde{c}}{(1-\tilde{l})}=(1-\beta)(1-\delta) \tilde{y} \\
\tilde{y}=\frac{\tilde{s}}{\beta}
\end{gathered}
$$

La solución del sistema anterior da lugar a los resultados de la proposición que se describen en los ítems c), d) y e). 


\section{Condiciones de primer orden para el problema (18)}

Por definición, las condiciones de primer orden para $\alpha$ y $\beta$ en el problema (18) determinan respectivamente que:

$$
\begin{aligned}
& \tau \frac{\partial U(0)}{\partial \alpha}+(1-\tau) \frac{\partial \widetilde{U}}{\partial \alpha}=0 \\
& \tau \frac{\partial U(0)}{\partial \beta}+(1-\tau) \frac{\partial \widetilde{U}}{\partial \beta}=0
\end{aligned}
$$

Por consiguiente, para resolver las ecuaciones (20) y (21), basta con encontrar las derivadas señaladas en (A.5) y (A.6). Usando la expresión para $U(0)$, tenemos que:

$$
\begin{gathered}
\frac{\partial U(0)}{\partial \alpha}=\left[\frac{\gamma(1-\delta)}{\beta(1-\gamma)}\right]^{\gamma(1-\sigma)} s 0^{\frac{\gamma \delta(1-\sigma)}{1-\delta}} \\
\frac{\partial U(0)}{\partial \alpha}=\left[\frac{\gamma(1-\delta)}{\beta(1-\gamma)}\right] s^{\frac{\gamma \delta(1-\sigma)}{1-\delta}} \\
\frac{\left(\frac{1-\sigma}{1-\delta}\right)\left[\phi(\alpha)^{\frac{1}{1-\delta}}-s_{0}^{\frac{1}{1-\delta}}\right]^{-\sigma} \phi(\alpha)^{\frac{(1-\gamma)(1-\sigma)}{1-\delta}} \phi^{\prime}(\alpha)\left[\gamma \phi(\alpha)^{\frac{1}{1-\delta}}+(1-\gamma) s_{0}^{\frac{1}{1-\delta}}\right]}{\phi(\alpha)^{2 \frac{(1-\gamma)(1-\sigma)}{1-\delta}}}+\frac{\varphi}{\alpha}
\end{gathered}
$$

Es fácil verificar que $\phi^{\prime}(\alpha)=-\eta \phi(\alpha) /(1-\alpha)$. Usando este hecho en la expresión anterior llegamos a:

$$
\frac{\partial U(0)}{\partial \alpha}=-\left[\frac{\gamma(1-\delta) s_{0}}{\beta(1-\gamma)}\right] \frac{\eta\left(\frac{1-\sigma}{1-\delta}\right)\left[\phi(\alpha)^{\frac{1}{1-\delta}}-s_{0} \frac{1}{1-\delta}\right]^{-\sigma}\left[\gamma \phi(\alpha)^{\frac{1}{1-\delta}}+(1-\gamma) s_{00}^{\frac{1}{1-\delta}}\right]}{(1-\alpha) \phi(\alpha)^{\frac{(1-\gamma)(1-\sigma)}{(1-\delta)}}}+\frac{\varphi}{\alpha}
$$

Por otro lado:

$$
\frac{\partial \widetilde{U}}{\partial \alpha}=\frac{\varphi}{\alpha}
$$

Sustituyendo (A.7) y (A.8) en (A.5), obtenemos la ecuación (20). Procediendo de la misma manera con respecto a $\beta$ tenemos que:

$$
\frac{\partial U(0)}{\partial \beta}=-\gamma \beta^{-1}\left[\frac{\gamma(1-\delta)^{\gamma(1-\sigma)}}{\beta(1-\gamma)} \text { so } \frac{\gamma \delta(1-\sigma)}{(1-\delta)} \frac{\left[\phi(\alpha)^{\frac{1}{(1-\delta)}}-s_{0}^{\frac{1}{(1-\delta)}}\right]^{1-\sigma}}{\phi(\alpha)^{\frac{(1-\gamma)(1-\sigma)}{(1-\sigma)}}}\right.
$$

También tenemos que:

$$
\begin{gathered}
\frac{\partial \widetilde{U}}{\partial \beta}=-\frac{\gamma(1-\sigma)(1-\gamma)^{(1-\gamma)(1-\sigma)} \beta^{-\gamma(1-\sigma)}(\tilde{s}-\beta \bar{g})^{(1-\sigma)}}{(1-\sigma)[(1-\gamma)(\tilde{s}-\beta \bar{g})+\gamma(1-\delta)(1-\beta) \tilde{s}]}+ \\
+\frac{(1-\gamma)^{(1-\gamma)(1-\sigma)} \beta^{\gamma(1-\sigma)}(1-\sigma)(\tilde{s}-\beta \bar{g})^{-\sigma}[(1-\sigma)(\tilde{s}-\beta \bar{g})+\delta \sigma(1-\beta) \tilde{s}]^{(1-\gamma)(1-\sigma)-1}}{(1-\sigma)} \times
\end{gathered}
$$




$$
\frac{\{[(1-\gamma) \delta \sigma \tilde{s}-\gamma(1-\sigma) \bar{g}](\tilde{s}-\beta \bar{g})-\delta \sigma(1-\beta) \tilde{s} \bar{g}\}}{[(1-\sigma)(\tilde{s}-\beta \bar{g})+\delta \sigma(1-\beta) \tilde{s}]^{2(1-\gamma)(1-\sigma)}}
$$

Sustituyendo (A.9) y (A.10) en (A.6), después de algunas manipulaciones algebraicas obtenemos la ecuación (21).

\section{Demostración de la proposición 2}

Proposición 2: Si $\eta>\left(\frac{1-\gamma}{\gamma}\right)^{\gamma(1-\sigma)} / \tau(1-\sigma)$ y $l(0)>(0,5)^{1-\delta}$, entonces $\widehat{\alpha}<0,5$.

\section{Demostración:}

Basta con probar que en las condiciones de enunciación, el lado izquierdo (22) es mayor que la unidad. Inicialmente, obsérvese que el término $\left[\frac{\tau \eta(1-\sigma)}{(1-\delta)}\right]\left[\frac{\gamma(1-\delta) s_{0}}{\widehat{\beta}(1-\gamma)}\right]^{\gamma(1-\sigma)}$ es también mayor que uno.

Esto ocurre debido a la suposición de que $\eta>\left(\frac{1-\gamma}{\gamma}\right)^{\gamma(1-\sigma)} / \tau(1-\sigma)^{\mathrm{y}}$ al hecho de que $\frac{s_{0}}{\beta}=y_{0}>1$. Por lo tanto, el resultado se establece si podemos probar que:

$$
\frac{\left[\phi(\alpha)^{\frac{1}{1-\delta}}-s_{0} \frac{1}{1-\delta}\right]^{-\sigma}\left[\gamma \phi(\widehat{\alpha})^{\frac{1}{1-\delta}}+(1-\gamma) s_{0}^{\frac{1}{1-\delta}}\right]}{\phi(\widehat{\alpha})^{\frac{(1-\gamma)(1-\sigma)}{(1-\delta)}}}>1
$$

Aplicando una reducción ad absurdum, asumamos que esta afirmación no es válida. En este caso, tenemos que:

$$
\left[\gamma \phi(\widehat{\alpha})^{\frac{1}{1-\delta}}+(1-\gamma) s_{0}^{\left.\frac{1}{1-\delta}\right]} \leq\left[\phi(\widehat{\alpha})^{\frac{1}{1-\delta}}-s_{0}^{\frac{1}{1-\delta}}\right]^{\sigma} \phi(\widehat{\alpha})^{\frac{(1-\gamma)(1-\sigma)}{1-\delta}}\right.
$$

Como $l(0)<1$ podemos fácilmente demostrar que $\varphi(\widehat{\alpha})>s_{0}$. Tras algunas manipulaciones, la desigualdad en (A.10) puede reescribirse como:

$$
s_{0}^{\frac{1}{1-\delta}}<\left[1-\left(\frac{s_{0}}{\phi(\widehat{\alpha})}\right)^{\frac{1}{1-\delta}}\right] \sigma(\widehat{\alpha})^{\frac{1-\gamma(1-\sigma)}{1-\delta}}
$$

o incluso:

$$
\left(\frac{s_{0}}{\phi(\widehat{\alpha})}\right)^{\frac{1}{1-\delta}}<\left[1-\left(\frac{s_{0}}{\phi(\widehat{\alpha})}\right)^{\frac{1}{1-\delta}}\right] \sigma(\widehat{\alpha})^{\frac{1-\gamma(1-\sigma)}{1-\delta}}
$$

Dado que $\frac{S 0}{\phi(\widehat{\alpha})}=I(0)$, la ecuación (A.11) puede ser reescrita como:

$$
l(0)^{\frac{1}{1-\delta}}<\left[1-l(0)^{\frac{1}{1-\delta}}\right]^{\sigma} \phi(\widehat{\alpha})^{\frac{-\gamma(1-\sigma)}{1-\delta}}
$$


Para $\sigma<l$, es evidente que $\left[1-l(0)^{\frac{1}{1-\delta}}\right]^{\sigma}<1-l(0)^{\frac{1}{1-\sigma}}$. En este caso, la ecuación (A.12)
implica que: $\frac{l(0)^{\frac{1}{1-\delta}}}{\left(1-l(0)^{\frac{1}{1-\delta}}\right)}<\phi(\widehat{\alpha})^{\frac{-\gamma(1-\sigma)}{1-\delta}}<\phi(\widehat{\alpha})^{\frac{-\gamma(1-\sigma)}{1-\delta}}$.

Dadas las condiciones de la proposición y usando la definición de $\phi(\alpha)$, tenemos que: $\frac{l(0)^{\frac{1}{1-\delta}}}{\left(1-l(0)^{\frac{1}{1-\delta}}\right)}<\phi(\widehat{\alpha})^{\frac{-\gamma(1-\sigma)}{1-\delta}}$. Esa contradicción produce el resultado deseado. 